ARTICLE

\title{
Fibril polymorphism affects immobilized non-amyloid flanking domains of huntingtin exon1 rather than its polyglutamine core
}

Hsiang-Kai Lin', Jennifer C. Boatz ${ }^{1}$, Inge E. Krabbendam², Ravindra Kodali, Zhipeng Hou ${ }^{3, \dagger}$, Ronald Wetzel ${ }^{1}$, Amalia M. Dolga², Michelle A. Poirier ${ }^{3} \&$ Patrick C.A. van der Wel ${ }^{1}$

Polyglutamine expansion in the huntingtin protein is the primary genetic cause of Huntington's disease (HD). Fragments coinciding with mutant huntingtin exon1 aggregate in vivo and induce HD-like pathology in mouse models. The resulting aggregates can have different structures that affect their biochemical behaviour and cytotoxic activity. Here we report our studies of the structure and functional characteristics of multiple mutant htt exon1 fibrils by complementary techniques, including infrared and solid-state NMR spectroscopies. Magic-angle-spinning NMR reveals that fibrillar exon1 has a partly mobile $\alpha$-helix in its aggregation-accelerating $\mathrm{N}$ terminus, and semi-rigid polyproline II helices in the proline-rich flanking domain (PRD). The polyglutamine-proximal portions of these domains are immobilized and clustered, limiting access to aggregation-modulating antibodies. The polymorphic fibrils differ in their flanking domains rather than the polyglutamine amyloid structure. They are effective at seeding polyglutamine aggregation and exhibit cytotoxic effects when applied to neuronal cells.

\footnotetext{
${ }^{1}$ Department of Structural Biology, University of Pittsburgh School of Medicine, 3501 Fifth Avenue, Pittsburgh, Pennsylvania 15213, USA. ${ }^{2}$ Department of Molecular Pharmacology, Groningen Research Institute of Pharmacy, University of Groningen, Antonius Deusinglaan 1, 9713 AV Groningen, The Netherlands. ${ }^{3}$ Division of Neurobiology, Department of Psychiatry, Children's Medical Surgical Center, Johns Hopkins University School of Medicine, 600 North Wolfe Street, Baltimore, Maryland 21287, USA. †Present address: Russell H. Morgan Department of Radiology and Radiological Science, Johns Hopkins University School of Medicine, Baltimore, Maryland 21287, USA. Correspondence and requests for materials should be addressed to P.C.A.v.d.W. (email: vanderwel@pitt.edu).
} 
H untington's Disease (HD) is the most prevalent example of a family of neurodegenerative diseases that have the abnormal expansion of a polyglutamine stretch (polyQ) as their primary genetic cause ${ }^{1}$. HD is a devastating and as-yet incurable disease in which the polyQ expansion occurs within the first exon of the huntingtin protein (htt exon1). As a result of protease activity or missplicing, N-terminal fragments of the mutant protein are generated, including the htt exon 1 segment. Misfolding, self-assembly and aggregation of these fragments lead to a gain of toxic function, which ultimately leads to neuronal death. The exact mechanism of toxicity remains uncertain, and different studies report diverging levels of toxicity (or lack thereof) for detectable htt exon1 aggregates, with some reporting an apparent lack of correlation between aggregate burden and toxicity ${ }^{2}$. However, it is increasingly recognized that cells contain different types of aggregates, including also fibrillar aggregates that are not as easily detected as large inclusions ${ }^{3-5}$. Such polymorphism is reminiscent of other amyloids $^{6,7}$, and is important, given that the toxicity of htt exon1 aggregates is known to depend on their structure ${ }^{8,9}$. Accordingly, toxicity-reducing mechanisms in vivo may induce the generation of aggregate species with reduced toxicity ${ }^{9-11}$, in parallel to protein homeostasis and clearance mechanisms that reduce aggregation.

The structural differences that underlie the polymorphism of htt exon1 aggregates remain uncertain. Prior studies have generally attributed them to the expanded polyQ domain, even in cases where low-resolution structural data may not unambiguously distinguish the polyQ and non-polyQ domains ${ }^{8,12}$. While the expanded polyQ domain forms the 'core' of the fibrillar aggregates ${ }^{13-17}$, it has become clear that non-polyQ 'flanking' domains (Fig. 1) have dramatic influences on the misfolding and aggregation pathways of htt exon1 and other polyQ proteins ${ }^{18-23}$. The highly conserved 17 -residue $\mathrm{N}$-terminal flanking segment $\left(\mathrm{htt}^{\mathrm{NT}}\right)$ is important for the native function of htt, but also initiates and accelerates aggregation of mutant htt exon1 (refs 18-21). On the other hand, the C-terminal prolinerich domain (PRD) reduces the innate aggregation propensity of the preceding polyQ domain by modulating its conformational ensemble ${ }^{24}$. These flanking domains also are targets for aggregation-modulating post-translational modifications (PTMs), chaperones and antibodies (Fig. 1a) ${ }^{25-32}$. However, not all exon1-binding proteins are effective at modulating aggregation. While MW7 and other PRD-binding proteins inhibit aggregate formation and cellular toxicity $28-30,33,34$, the PRD-binding MW8 antibody does not ${ }^{30,35}$.

To understand exon1 aggregate polymorphism, the exon1 aggregation mechanism, and how both can be modulated by htt exon1-binding proteins and PTMs, it is crucial to know the structure of the aggregated species. We have been using magicangle-spinning (MAS) solid-state NMR (ssNMR) to study mutant htt exon 1 and shorter htt-derived peptide fibrils ${ }^{13,15,17,32}$. MAS ssNMR is a powerful tool for elucidating the structure of amyloid fibrils, and is the gold standard for identifying differences among polymorphic amyloid structures ${ }^{7,36}$. Mutant htt exon1 fibrils feature a well-defined amyloid core, consisting of polyQ $\beta$-hairpins ${ }^{17}$, while the flanking domains lack $\beta$-structure ${ }^{14-17}$. In fibrils formed by synthetic htt N-terminal fragments (HNTFs) that behave similar to full-length exon1 (ref. 37), the htt ${ }^{\mathrm{NT}}$ segment features a short amphipathic $\alpha$-helix ${ }^{13,15,32}$. Here we refer to these $\mathrm{htt}^{\mathrm{NT}} \mathrm{Q}_{30} \mathrm{P}_{10} \mathrm{~K}_{2}$ peptides (Fig. 1b) as HNTFs. A recent ssNMR study on fibrils prepared using thioredoxin-fused htt exon 1 failed to detect the signals for an $\alpha$-helical htt ${ }^{\mathrm{NT}}$, raising the possibility that $\mathrm{htt}^{\mathrm{NT}}$ has a different structure in fibrillar exon1 (ref. 16). This is an important issue, as the presence of $\alpha$-helical htt ${ }^{\mathrm{NT}}$ provided support for the idea that $\alpha$-helical htt ${ }^{\mathrm{NT}}$ segments play a critical role in exon1 oligomerization and aggregation $^{13,15,20,21,32}$. Thus, prior studies of htt exon1 fibrils have been unable to offer a consistent picture of either the detailed structure of the fibrils (and in particular the flanking domains) or the origins of reported fibril polymorphism. Here we report MAS ssNMR studies of different fibrils prepared from htt exon1 featuring a disease-relevant 44-residue polyQ domain. We find that the htt ${ }^{\mathrm{NT}}$ also in exon1 fibrils contains a partly immobilized $\alpha$-helix, and probe in detail the structure and dynamics of the C-terminal flanking domain. The polyQproximal region of the $\mathrm{PRD}$ is immobilized near the amyloid core surface, reducing access to PRD-binding antibodies. An obvious and reproducible temperature-dependent amyloid-like fibril polymorphism affecting the cytotoxic aggregates is reflected

a

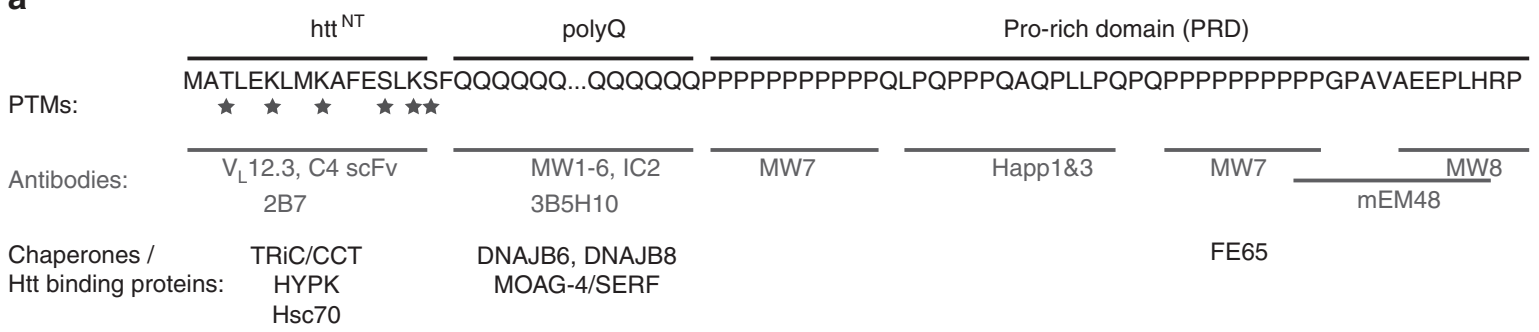

b

$$
\text { HNTF peptide htt }{ }^{\mathrm{NT}} \mathrm{Q}_{30} \mathrm{P}_{10} \mathrm{~K}_{2}: \quad \frac{\text { MATLEKLMKAFESLKSFQQQQQQ...QQQQQQPPPPPPPPPPKK }}{\mathrm{htt}^{\mathrm{NT}}} \frac{\text { OligoPro }}{\text { polyQ }}
$$

C

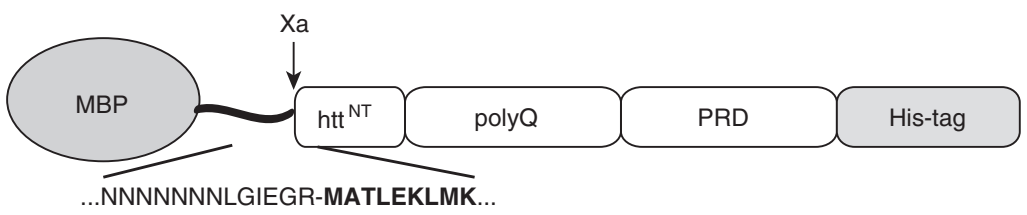

Figure 1 | Htt exon1 sequence and domain structure. (a) The domain structure and sequence of htt exon1 is shown at the top. The locations of PTMs,

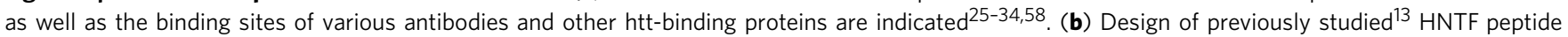
$h t{ }^{N T} \mathrm{Q}_{30} \mathrm{P}_{10} \mathrm{~K}_{2}$. (c) Design of the MBP fusion protein, with the sequence of the Factor Xa cleavage site in the linker shown below. 
in detectable changes, not in the polyQ as previously suggested, but rather in the non-amyloid flanking domains.

\section{Results}

Fibril formation by disease-relevant mutant htt exon1. For in vitro studies, mutant htt exon 1 is usually expressed as a fusion protein in which the $\mathrm{N}$ terminus of exon1 is fused to a soluble protein tag to inhibit aggregation ${ }^{38-40}$. Cleavage of the linker releases exon1, but commonly leaves behind a non-native $\mathrm{N}$ terminus ${ }^{30,38-40}$. Such modifications of htt ${ }^{\mathrm{NT}}$ can modify the aggregation and toxicity of htt exon 1 (refs 8,18), similar to the effects of htt ${ }^{\mathrm{NT}}$ mutations and $\mathrm{PTMs}{ }^{20,32}$. We therefore generated a fusion construct for htt exon1 with a 44-residue polyQ domain that produces an $\mathrm{N}$ terminus just as it is encoded in the genome when the fusion partner is cleaved ${ }^{15}$. A 10-residue linker segment was eliminated from a previously used maltose-binding protein (MBP) fusion protein construct ${ }^{40}$ to yield a new Factor Xa cleavage site (IEGR-MATL) designed to generate the desired 17-residue htt ${ }^{\mathrm{NT}}$ (Fig. 1c). To test for correct cleavage, we performed SDS-PAGE and mass spectrometry analyses. Efficient protease cleavage is observed (Fig. 2a), resulting in release of MBP and htt exon1 with the expected molecular masses (Supplementary Table 1 and Supplementary Fig. 1). Thus, even without the extended linker the cleavage site is easily proteaseaccessible.

Using transmission electron microscopy (TEM) we observed the aggregation of the released htt exon1 (ref. 17). Across a series of independent aggregation trials we observed mature aggregates that appear as elongated unbranched amyloid-like fibrils, but differed specifically in the fibre widths. Consistent with prior work $^{8}$, we find that the temperature at which the aggregation occurs plays a critical role in dictating the fibril morphology. At $37^{\circ} \mathrm{C}$, narrow fibrils are formed (Fig. $2 \mathrm{~b}$ and Supplementary Fig. 2a,b). Aggregation at $22^{\circ} \mathrm{C}$ yields larger fibril widths (Fig. $2 \mathrm{c}$ and Supplementary Fig. 2c,d). By Fourier transform infrared spectroscopy (FTIR) both fibril types (Fig. 2d,e) share the same dominant features previously reported for other polyQ, HNTF and htt exon1 fibrils (for example, Fig. $2 \mathrm{f}$ ) $^{8,13,37,41,42}$. The two fibril species display a few differences in their less prominent signals (arrows in Fig. 2e). The partly overlapping resonance frequencies expected for $\alpha$-helices, $\beta$-sheet polyQ amyloid and PPII-helical oligoproline (oligoPro) are indicated in Fig. $2 \mathrm{~g}$ (refs 13,37,41-44). The differentiating FTIR signals show most similarity to signals expected for the PRD, but an unambiguous assignment is challenging. These polymorphic TEM and FTIR features are reproduced in independently prepared samples (for example, Supplementary Fig. 3).

SSNMR shows no evidence of polymorphism in the amyloid core. To analyse the fibril structure in more detail, we applied MAS ssNMR to uniformly ${ }^{13} \mathrm{C}$ and ${ }^{15} \mathrm{~N}\left(\mathrm{U}_{-}{ }^{13} \mathrm{C},{ }^{15} \mathrm{~N}\right)$-labelled htt exon1 fibrils (see Methods and Supplementary Table 2 for experimental details). To identify the rigid domains we use experiments reliant on cross-polarization (CP) and other dipolar-coupling-based transfers, which filter out highly mobile residues. Figure $3 a-c$ compares the $1 \mathrm{D}{ }^{13} \mathrm{C} \mathrm{CP}$ spectra of htt exon1 fibrils prepared at 22 and $37^{\circ} \mathrm{C}$. The spectra are essentially indistinguishable, with no indication of substantial structural differences in the immobile parts of the fibrils. We gain more insights into these rigid domains using two-dimensional (2D) spectra that afford sitespecific resolution and thus assignment of residues or residue types (see Supplementary Table 3 ). Figure 4 shows a $2 \mathrm{D}{ }^{13} \mathrm{C}-{ }^{13} \mathrm{C}$ spectrum obtained with $\mathrm{CP}$ and dipolar-assisted rotational resonance recoupling $(\mathrm{CP} / \mathrm{DARR})$. This spectrum is dominated by signals from the polyQ amyloid core (boxed), with its highly characteristic resonance frequencies ${ }^{13,15-17,45,46}$. A detailed analysis of $2 \mathrm{D}$ spectra for the htt exon 1 fibrils prepared at 22 and $37^{\circ} \mathrm{C}$ (refs 15,17) reveals no detectable differences in terms of the Gln chemical shifts, relative peak heights, crosscorrelation patterns or dynamics (see also below). This stands in contrast to polymorphic $A \beta$ and $\alpha$-synuclein fibrils that are easily distinguished by their ssNMR spectral differences indicative of distinct amyloid core structures ${ }^{7,36}$, as well as prior reports of significant structural changes affecting the polyQ core itself ${ }^{8,12}$. a

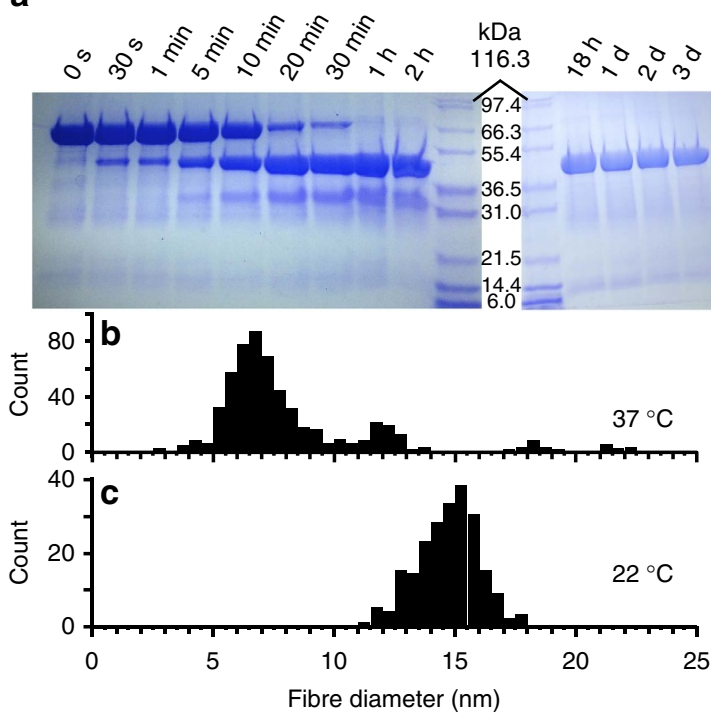

d

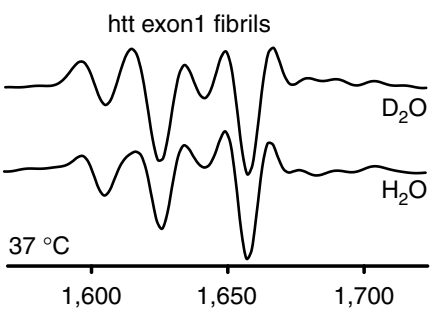

e

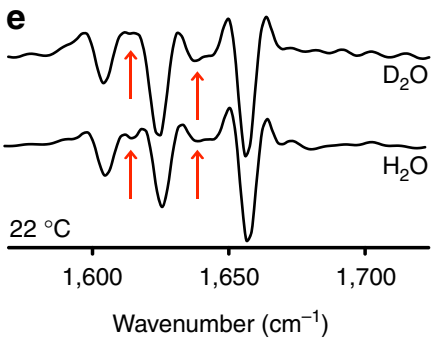

f

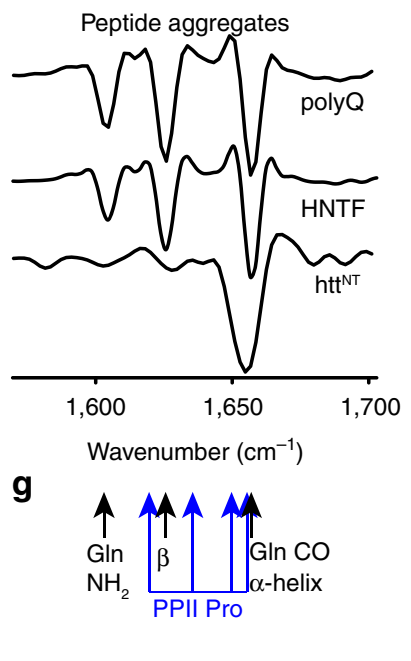

Figure 2 | Cleavage and aggregation of mutant htt exon1. (a) SDS-PAGE gels showing time-dependent factor Xa cleavage at $22^{\circ} \mathrm{C}$. (b,c) Fibril width derived from negative-stain TEM on the mature fibrils formed at $37^{\circ} \mathrm{C}\left(597\right.$ measurements over 99 fibrils) and $22^{\circ} \mathrm{C}(219$ measurements over 73 fibrils). (d) Second-derivative FTIR of htt exon1 fibrils formed at $37^{\circ} \mathrm{C}$ and $(\mathbf{e}) 22^{\circ} \mathrm{C}$, for fibrils dispersed in either $\mathrm{H}_{2} \mathrm{O}$ or $\mathrm{D}_{2} \mathrm{O}$. The coloured arrows mark the most notable differences between the fibril types. (f) Reference data on fibrillar $\mathrm{K}_{2} \mathrm{Q}_{31} \mathrm{~K}_{2}, \mathrm{HNTF}\left(\mathrm{htt}^{\mathrm{NT}} \mathrm{Q}_{30} \mathrm{P}_{10} \mathrm{~K}_{2}\right.$ ) fibrils, and aggregated $\alpha$-helical htt ${ }^{\mathrm{NT}}$ in PBS buffer, adapted with permission from ref. 13, Copyright 2011 American Chemical Society. (g) Resonance frequencies of different secondary structure elements. 


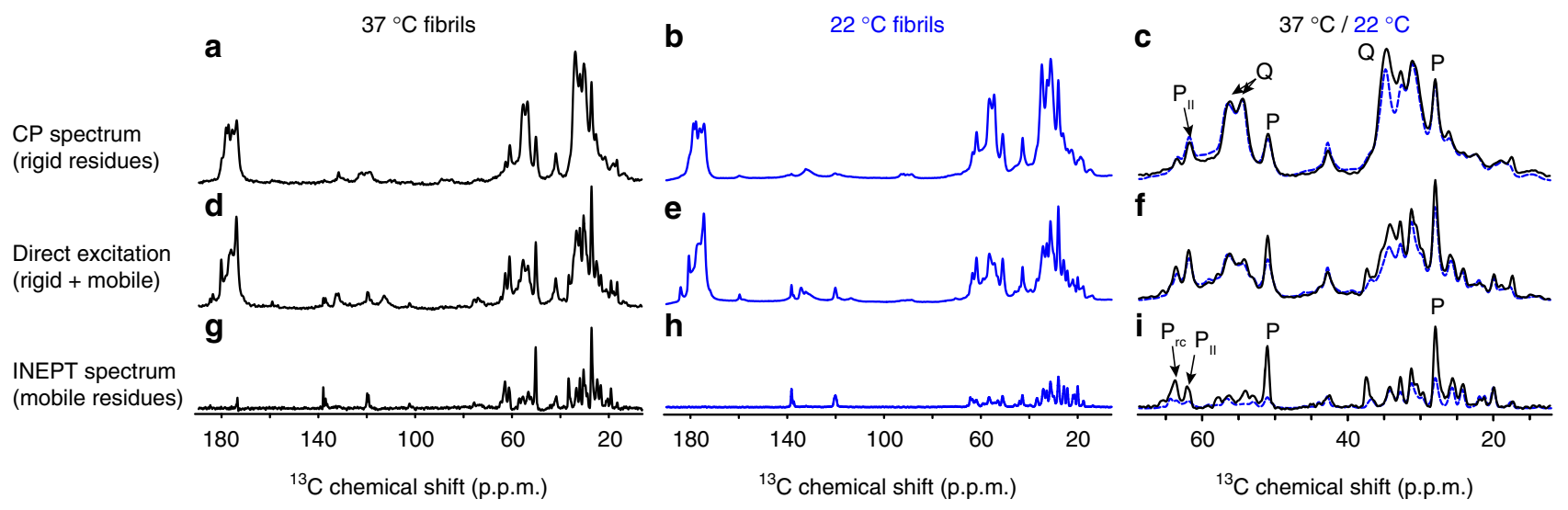

Figure 3 | 1D ${ }^{\mathbf{1 3}} \mathbf{C}$ ssNMR spectra of uniformly ${ }^{\mathbf{1 3}} \mathbf{C}$ - and ${ }^{\mathbf{1 5}} \mathbf{N}$-labelled htt exon1 fibrils. (a,d,g) Fibrils were formed at $37^{\circ} \mathrm{C}$, or $(\mathbf{b}, \mathbf{e}, \mathbf{h}) 22^{\circ} \mathrm{C}$, and studied using (a-c) cross-polarization (rigid residues), (d-f) direct polarization and (g-i) INEPT-based (mobile residues) MAS ssNMR. (c,f,i) Overlaid aliphatic regions, with assignments indicating the random coil $\left(P_{r c}\right)$ and PPII-helical Pro $\left(P_{\|}\right)$. The NMR measurements were performed at $275 \mathrm{~K}$ on a $600 \mathrm{MHz}$ ( ${ }^{1} \mathrm{H}$ frequency) spectrometer.

Flanking domains feature immobilized $\alpha$ - and PPII helices. The 2D CP/DARR spectrum also includes peaks (underlined labels in Fig. 4a) that reproduce the $\alpha$-helical htt ${ }^{\mathrm{NT}}$ signals seen previously in the HNTF fibrils (Fig. 4b $)^{13,15}$. The observed resonance frequencies identify a localized $\alpha$-helix, based on the dependence of $C \alpha$ and $C \beta$ chemical shift $\delta$ on the backbone structure. In Fig. $4 \mathrm{c}, \mathrm{d}$, this is visualized as positive 'secondary shifts,' $\Delta \delta(\mathrm{C} \alpha-\mathrm{C} \beta)$, for the $\alpha$-helix spanning residues $4-11$ of htt ${ }^{\mathrm{NT}}$ (blue bars). The one-dimensional (1D) and 2D CP spectra also feature peaks from the PRD. The dominant PRD signals in Fig. 4a are for Pro residues with chemical shifts characteristic of PPII helices, as previously seen in HNTF and htt exon1 fibrils ${ }^{15,16}$. A weaker, but still strong, second set of Pro signals is observed with chemical shifts resembling those of Pro in intrinsically disordered proteins (IDPs), indicating a randomcoil-like $\left(\right.$ Pro $\left._{\mathrm{RC}}\right)$ structure ${ }^{47} \cdot{ }^{13} \mathrm{C}$ direct-excitation spectra indicate an $\sim 2: 1$ ratio of the two populations of Pro residues, independent of the fibril formation temperature (Fig. $3 \mathrm{~d}-\mathrm{f}$ and Supplementary Fig. 4). Other, non-Pro, PRD signals are visible, including peaks for the unique Gly and Val residues G100 and $\mathrm{V} 103$. The fact that these $\mathrm{htt}^{\mathrm{NT}}$ and PRD residues are visible in CP-based spectra implies that both flanking domains are partly immobilized by interactions with the amyloid core or with each other.

Restricted motion of the polyQ-proximal flanking segments. $\mathrm{CP}$-visible residues may be immobilized, but can nonetheless feature significant and detectable dynamics ${ }^{15}$. Evidence of such dynamics was obtained in a series of ${ }^{13} \mathrm{C}-{ }^{13} \mathrm{C}$ recoupling experiments with proton-driven spin diffusion (PDSD) times of 0-500 ms (Supplementary Fig. 5a-f). The transfer or buildup of polarization (or signal) in such experiments is dependent on dipolar couplings between nearby ${ }^{13} \mathrm{C}$ and ${ }^{1} \mathrm{H}$ atoms. Dynamics cause an apparent reduction in these couplings, leading to slower and weaker polarization transfer profiles as illustrated in Fig. 5a. In a rigid crystalline peptide, directly bonded $\mathrm{C} \alpha-\mathrm{C} \beta$ carbons show a fast polarization buildup that reaches a $10-20 \%$ polarization transfer within the first $10-20 \mathrm{~ms}$ (solid lines). Some variations in the polarization transfer are typical of the complex mechanism underlying PDSD recoupling ${ }^{48}$. Intermediate timescale molecular motion reduces the effective dipolar couplings and increases relaxation, causing a reduction in both the transfer rate and the transfer maximum ${ }^{15}$. Fully dynamic molecules, such as those in solution, experience complete averaging of the dipolar couplings and, therefore, a lack of
${ }^{13} \mathrm{C}-{ }^{13} \mathrm{C}$ transfer (dashed line). Thus, these PDSD buildup profiles can be used to detect dynamics.

In the exon1 fibrils, we observe a fast buildup and high maximum for $C \alpha-C \beta$ peaks of the amyloid core Gln (Fig. $5 b$ ) that indicates a crystal-like rigid structure. Small variations among the curves are most likely explained by the complex PDSD mechanism rather than changes in dynamics. Much larger changes are seen in Fig. $5 c$, which shows the buildup curves for the flanking domain Ala signals. Compared to the Gln, the buildup is slower and the attained maximum is much reduced. These dramatic differences in the PDSD buildup curves can only be explained by motion-induced reductions of the dipolar couplings and increases in relaxation. In the PRD, we see that both types of Pro (PPII and random coil) have one-bond signal transfer (Fig. 4d) that is both lower and slower than that of the amyloid core (Fig. 4b). Two-bond $\mathrm{C} \alpha-\mathrm{C} \gamma$ transfers reveal a difference between IDP-like and PPII Pro (Supplementary Fig. 5g), which indicates that the former undergo increased dynamics. As a secondary probe of these dynamics, we also measured the motional averaging of ${ }^{1} \mathrm{H}-{ }^{13} \mathrm{C} \alpha$ dipolar couplings for the Pro and Gln residues, using a dipolar-chemical shift (DIPSHIFT) experiment ${ }^{15,49}$. The results of these experiments match the results of the PDSD-based measurements. Unlike the dipolar oscillations of the rigid Gln backbones, the Pro residues experience attenuated ${ }^{1} \mathrm{H}-{ }^{13} \mathrm{C}$ dipolar couplings (Supplementary Fig. 6). The PRDs of fibrils formed at $22^{\circ} \mathrm{C}$ appear to be slightly more dynamic compared to the $37^{\circ} \mathrm{C}$ fibrils. Thus, Pro residues in the flanking domains in htt exon1 fibrils have an intermediate timescale dynamic behaviour similar to those of the htt ${ }^{\mathrm{NT}}$ $\alpha$-helices ${ }^{15}$.

Variable dynamics of the PRD flanking domains. CP-based ssNMR spectra of the polymorphic fibrils showed no chemical shift differences in the polyQ amyloid core or immobilized parts of the flanking domains; however, there is evidence of motional differences in the latter. To test for differences in more dynamic parts of the fibrils, we turn to a different set of motion-sensitive ssNMR experiments. Solution-NMR-like INEPT (Insensitive Nuclei Enhanced by Polarization Transfer) spectra require nuclei to experience slow ${ }^{1} \mathrm{H} \quad \mathrm{T}_{2}$ relaxation in absence of ${ }^{1} \mathrm{H}-{ }^{1} \mathrm{H}$ decoupling, and thus show only residues with very high mobility. Both the 22 and $37^{\circ} \mathrm{C}$ fibrils yield peaks in INEPT spectra (Fig. 3g-i). Strikingly, we observe a lot more signal for fibrils formed at $37^{\circ} \mathrm{C}$ compared to those obtained at $22^{\circ} \mathrm{C}$. Both exon 1 samples differ from htt ${ }^{\mathrm{NT}} \mathrm{Q}_{30} \mathrm{P}_{10} \mathrm{~K}_{2}$ fibrils, which show no INEPT 

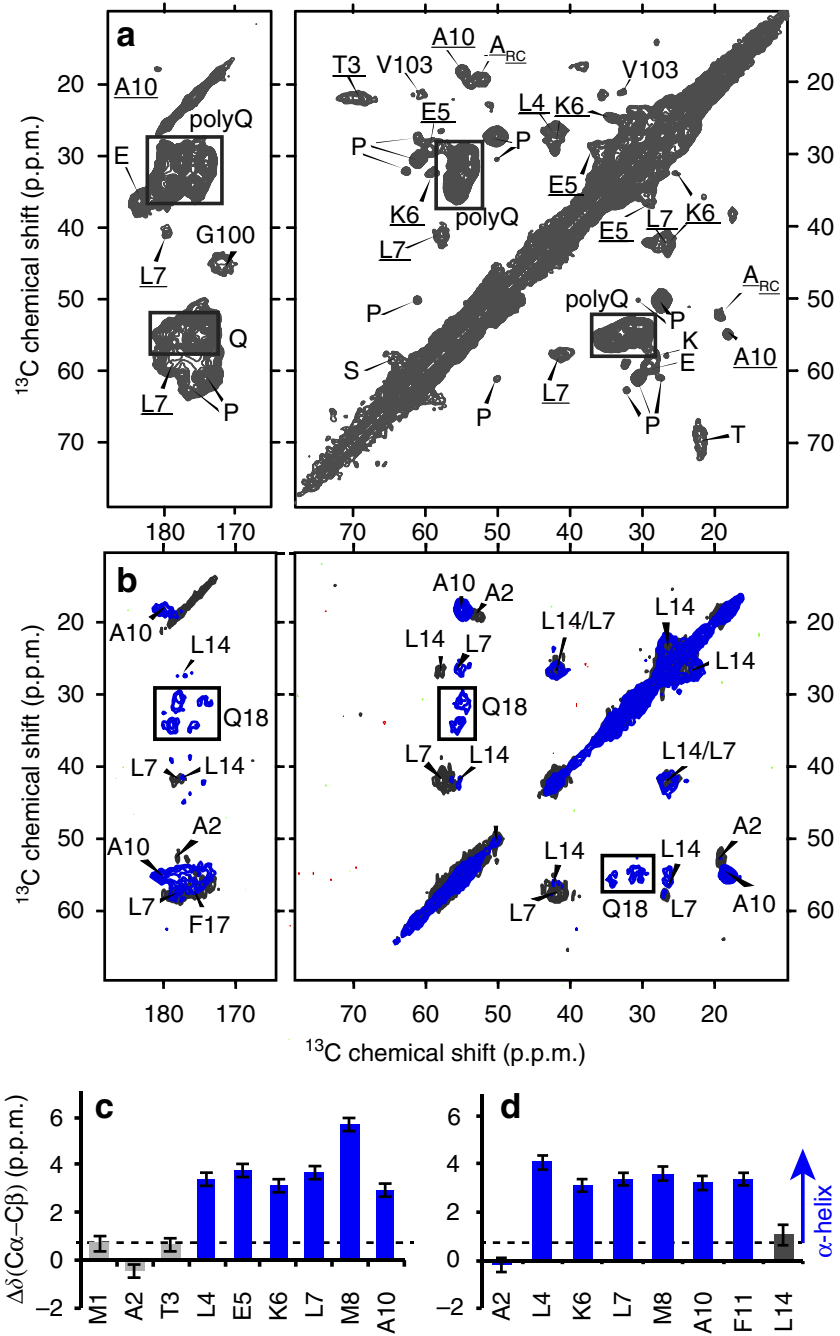

Figure 4 | MAS ssNMR identifies the immobilized parts of htt exon1 fibrils. (a) $2 \mathrm{D}^{13} \mathrm{C}-{ }^{13} \mathrm{C} C P / D A R R$ spectrum on $\mathrm{U}^{13} \mathrm{C}^{15} \mathrm{~N}$ htt exon 1 fibrils prepared at $22^{\circ} \mathrm{C}$. Signals from the poly $\mathrm{Q}$ domain are boxed, and underlined peak assignments are from residues in $\mathrm{htt}^{\mathrm{NT}}$. (b) Analogous 2D spectra of HNTF fibrils with site-specific $\mathrm{U}_{-13}^{13} \mathrm{C}_{1}^{15} \mathrm{~N}$-labelling of the indicated $\mathrm{htt}{ }^{\mathrm{NT}}$ residues and the first $\mathrm{Gln}$ of the $\mathrm{Q}_{30}$ repeat $(\mathrm{Q} 18)^{13}$. The blue and black contours are for samples labelled in residues A10/F11/L14/Q18, or A2/L7/ F17, respectively. (c,d) Detection of $\alpha$-helical secondary structure (blue bars) in htt ${ }^{\mathrm{NT}}$ residues of htt exon1 (c) and HNTF (d) fibrils, based on the secondary chemical shifts $\Delta \delta(C \alpha-C \beta)$. Error bars reflect the s.d. in the chemical shift (see Supplementary Table 3). The NMR measurements were performed at $267-275 \mathrm{~K}$ on a $600 \mathrm{MHz}\left({ }^{1} \mathrm{H}\right)$ spectrometer. Panel $(\mathbf{b})$ was adapted with permission from ref. 13, Copyright 2011 American Chemical Society.

signals, suggesting that the dynamic residues are in the latter part of the PRD that is missing from such peptides ${ }^{13,15,16}$. In 2D ${ }^{13} \mathrm{C}-{ }^{13} \mathrm{C}$ INEPT/TOtal through Bond correlation Spectroscopy (TOBSY) spectra for both fibril types (Fig. 6) peaks are observed that differ from those in the 2D CP-based spectra (see overlay in Supplementary Fig. 7), with a few exceptions discussed below. On the basis of the $2 \mathrm{D}$ data, we can assign the mobile residues or residue types, as marked in Figs 3 and 6 . The strongest peaks are from Pro residues (for example, Fig. 3i), with other peaks reflecting residue types that are only present in the tail end of the PRD: Val103, E105, E106 and Arg110. The chemical shifts indicate that this highly mobile C-terminal tail is unstructured.

As noted, the efficiencies of CP and INEPT-based spectra have opposite dependencies on molecular motion. Mobile residues visible as strong peaks in INEPT spectra are expected to be missing from $\mathrm{CP}$-based spectra. A residue with a prominent $\mathrm{CP}$ signal, in contrast, must be in some way immobilized, have fast $\mathrm{T}_{2}$ relaxation and thus be invisible in INEPT spectra. Seeing substantial peaks from the same residue in both spectra (Supplementary Fig. 7) implies that such a residue is present in different subpopulations that have markedly different mobilities. Intriguingly, this applies to many of the PRD signals: the PPII Pro, the IDP-like Pro, as well as V103. Overall, $\sim 2 / 3$ of the Pro are in PPII structure (Supplementary Fig. 4a,b). However, among the INEPT-visible mobile prolines it is the IDP-like peaks that are dominant (Supplementary Fig. 4c). We conclude that different molecules throughout the sample have PRDs with different degrees of flexibility. When comparing the fibril polymorphs, in the $37^{\circ} \mathrm{C}$ fibrils the population of proteins with dynamic PRDs is notably larger, even though the overall ratio of PPII to unstructured Pro remains the same (Supplementary Fig. 4a,b).

Polymorphic differences in PRD motion and accessibility. As protein dynamics often correlate to solvent interactions, we submitted both fibril forms to ssNMR measurements that can evaluate solvent exposure in a residue-specific manner. The employed experiments first eliminate the ${ }^{1} \mathrm{H}-{ }^{13} \mathrm{C} \mathrm{CP}$ signals generated from proteinaceous hydrogens by filtering out the latter based on their faster $\mathrm{T}_{2}$ relaxation. Then, we detect the signal recovery as a function of time-dependent transfer of solvent ${ }^{1} \mathrm{H}$ polarization into the fibril. Residues that are most solventexposed recover the fastest, while those that are buried take longer to re-appear. Figure 7a,b compares the overall, unfiltered $\mathrm{CP}$ signal (grey line) to the partly repolarized fibril signal after $7 \mathrm{~ms}$ of ${ }^{1} \mathrm{H}-{ }^{1} \mathrm{H}$ diffusion (blue), with both spectra normalized to their maximum peaks. In the $37^{\circ} \mathrm{C}$ fibrils the highest signal recovery is seen for Pro residues, indicating a high degree of solvent accessibility for the PRDs. The repolarization is fastest for the IDP-like Pro, consistent with their higher mobility. The data for the $22^{\circ} \mathrm{C}$ fibrils are different, in that the polarization transfer to the PPII Pro trails that of other parts of the fibrils. Thus, in these samples the PPII helices have a surprisingly reduced solvent accessibility, consistent with their more restricted motion. Conversely, the signals from $\mathrm{htt}^{\mathrm{NT}}$ are notably enhanced (upon $7 \mathrm{~ms}{ }^{1} \mathrm{H}-{ }^{1} \mathrm{H}$ transfer) compared to the $37^{\circ} \mathrm{C}$ fibrils, reminiscent of the htt ${ }^{\mathrm{NT}} \alpha$-helix in HNTF fibrils ${ }^{13,15}$. Thus, the flanking domains in these polymorphic fibrils feature correlated differences in their dynamics and solvent exposure.

Occlusion of the PRD domains limits antibody access. Next, we examined a number of biochemical or functional characteristics of the htt exon1 fibril polymorphs. First, dot blot experiments were used to probe the domain-specific accessibility of the fibrils to htt-exon1-specific antibodies ${ }^{35}$ (Fig. 7c). In line with prior reports ${ }^{30,35}$, exon1 fibril formation causes region-specific reductions in the binding of antibodies to their epitopes. PolyQbinding MW1 antibodies bind monomers, but have very low affinity for fibrils (Fig. 7c), because of complete sequestration of their epitopes ${ }^{30}$. The epitopes of the MW7 and MW8 antibodies are in the oligoPro segments and C-terminal PRD tail, respectively (Fig. 1a) ${ }^{35}$. MW8 binds to the PRDs of our mature exon1 fibrils, with an efficiency that is similar to the unaggregated protein (Fig. 7c). MW7 shows a reduced affinity to the aggregates, with the largest reduction in binding seen for the wider $22^{\circ} \mathrm{C}$ fibrils, which suggests an increased sequestration of its epitopes in those aggregates. These findings are consistent with prior studies that probed aggregated mutant htt exon1 with these antibodies, both in vitro and as cellular inclusions ${ }^{30,35}$. 

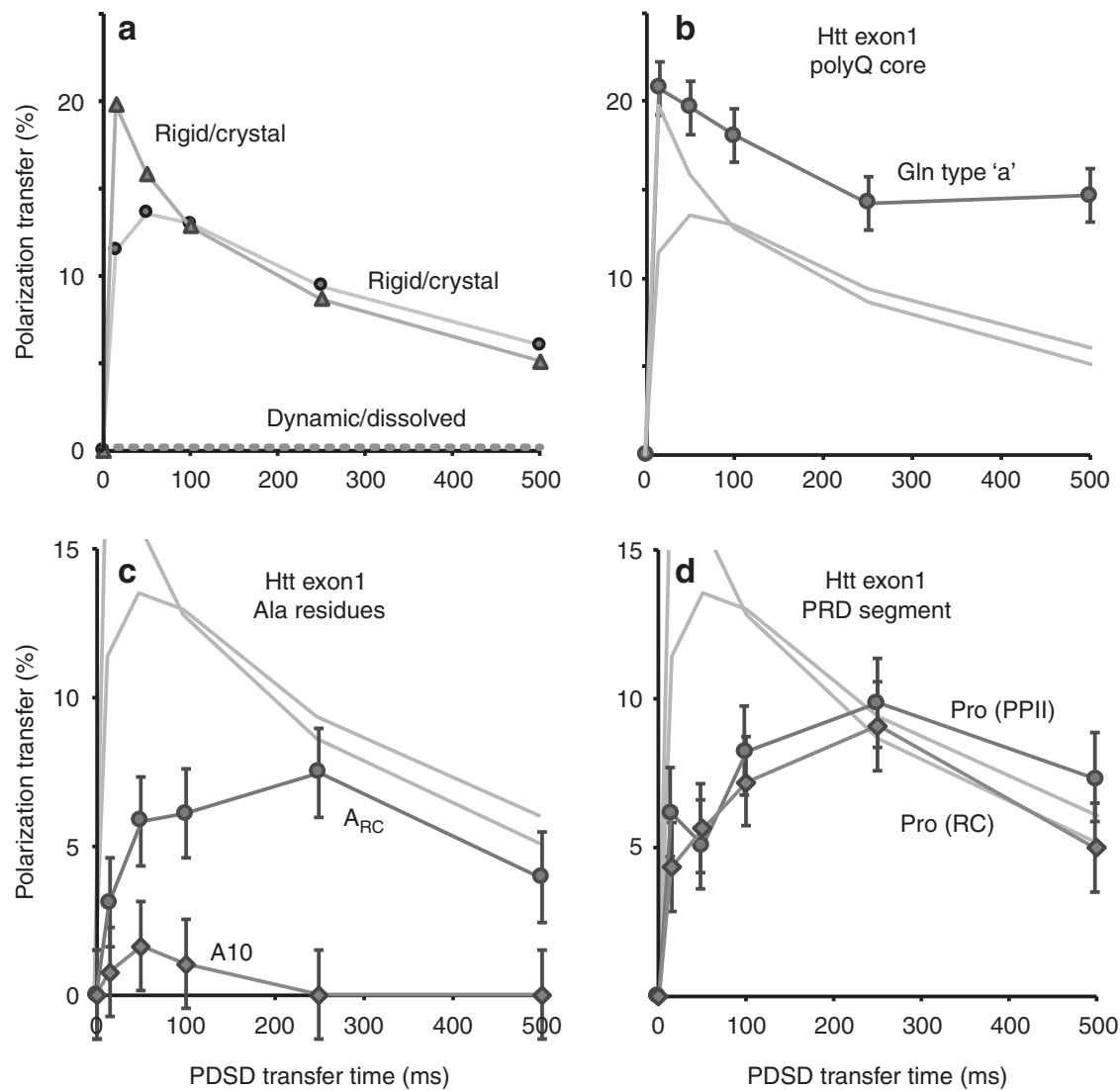

Figure 5 | SSNMR dipolar recoupling curves reveal exon1 fibril domain motion. (a) Reference PDSD buildup profiles for one-bond C $\alpha$-C $\beta$ cross-peaks of the crystalline dipeptide $\mathrm{N}$-acetyl-Val-Leu, reflecting an example of a fully rigid molecule. The dashed line illustrates the lack of buildup for a fully mobile (for example, dissolved) molecule. Intermediate motion is expected to lead to build-up curves in-between these extremes. (b) PDSD buildup profiles for $\mathrm{C} \alpha$-C $\beta$ peaks of type-' $\mathrm{a}^{\prime} \mathrm{G} \ln$ in the polyQ core, $(\mathbf{c}) \alpha$-helical A10 in htt ${ }^{\mathrm{NT}}$, random coil Ala $\left(\mathrm{A}_{\mathrm{RC}}\right)$ and (d) the random coil (RC) and PPII-structured Pro in the PRD of htt exon1 fibrils formed at $22^{\circ} \mathrm{C}$. Pale grey lines show the reference curves from a. Error bars indicate the s.d., as described in the Methods. The NMR measurements were performed at $275 \mathrm{~K}$ on a $600 \mathrm{MHz}\left({ }^{1} \mathrm{H}\right)$ spectrometer.

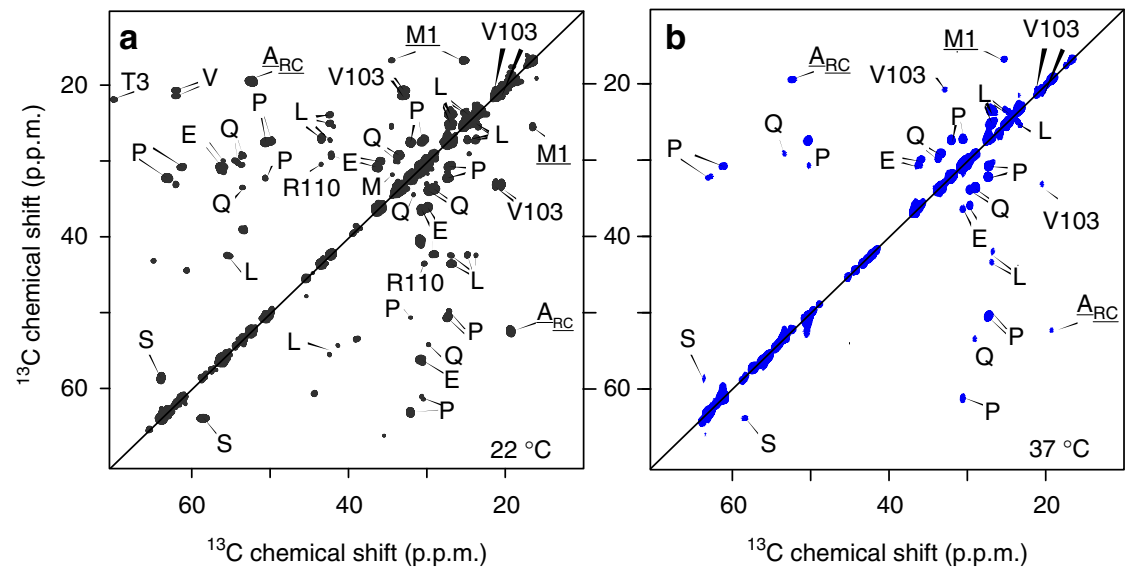

Figure 6 | Dynamic residues in the polymorphic htt exon1 fibrils identified via 2D INEPT-based ssNMR. ${ }^{13} \mathrm{C}-{ }^{13} \mathrm{C}$ INEPT/TOBSY spectra for fibrils prepared at $22^{\circ} \mathrm{C}(\mathbf{a})$, and $37^{\circ} \mathrm{C}(\mathbf{b})$. Observed residue types are from the very $\mathrm{C}$-terminal tail of the PRD, indicating that this part of the fibrillized protein is highly dynamic. Spectra acquired at $600 \mathrm{MHz}\left({ }^{1} \mathrm{H}\right)$ and $8.33 \mathrm{kHz}$ MAS, at a temperature of $275 \mathrm{~K}$.

Seeding activity and cytotoxic effects of the polymorphs. We also compared the seeding activity of two polymorphs using a previously reported seeding assa ${ }^{50}$. Figure 8 shows the results of this assay in which aggregation at $22^{\circ} \mathrm{C}$ in presence and absence of $20 \mathrm{~mol}-\%$ of pre-aggregated seeds was monitored by thioflavin $\mathrm{T}$ (ThT) fluorescence and HPLC-based monomer concentration measurements. In absence of seeds, aggregation (after trypsin cleavage) initiates with a lag phase that exceeds $4 \mathrm{~h}$ (Fig. 8b,d).
The presence of seeds abolishes the lag, leading to a significant decrease in the half time of aggregation. This seeding ability affects expanded polyQ proteins (Fig. 8) and shorter peptides with a 23-residue polyQ domain (Supplementary Fig. 8).

To probe for potential cytotoxic effects, we exposed two neuronal cell types to micromolar concentrations of each polymorph. Two different neuronal cell types were tested: an immortalized murine hippocampal neuronal cell line and human 
a

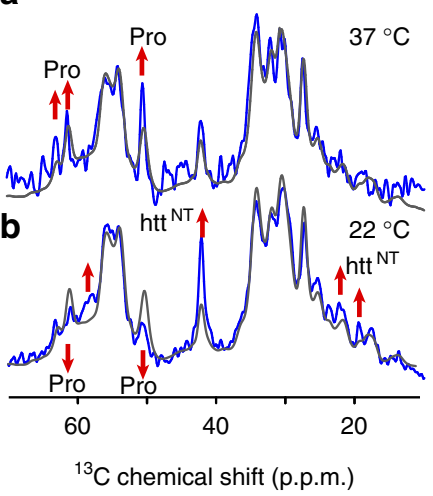

C

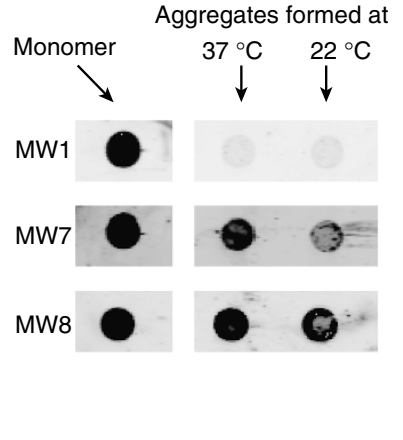

Figure 7 | Accessibility of the htt exon1 fibril PRDs probed by solventfiltered ssNMR and antibody binding. (a,b) Solvent accessibility of htt exon1 fibrils prepared at (a) $37^{\circ} \mathrm{C}$ and (b) $22^{\circ} \mathrm{C}$ probed by ssNMR. Peak intensities after $7 \mathrm{~ms}{ }^{1} \mathrm{H}-{ }^{1} \mathrm{H}$ diffusion from the solvent into the fibrils (blue) are compared to the ${ }^{13} \mathrm{C} \mathrm{CP}$ spectrum in absence of $\mathrm{T}_{2}$-based solvent filtering (grey). Each spectrum was normalized to the highest peaks to highlight the relative solvent exposures. Up/down arrows indicate sites with high/low solvent accessibility. The NMR measurements were performed at $275 \mathrm{~K}$ on a $600 \mathrm{MHz}\left({ }^{1} \mathrm{H}\right)$ spectrometer. (c) Dot blot analysis shows that in the monomeric protein the polyQ domain, oligoPro segments and PRD tail are all accessible for binding by MW1, MW7 and MW8, respectively (Fig. 1). Upon aggregation at 22 or $37^{\circ} \mathrm{C}, \mathrm{MW} 1$ binding to the polyQ is largely abolished, while the PRD tail is still strongly recognized by MW8. OligoPro binding by MW7 is weaker in the $22^{\circ} \mathrm{C}$ fibrils compared to the $37^{\circ} \mathrm{C}$ polymorph.

differentiated dopaminergic neurons ${ }^{51-53}$. Fibrils were applied at different concentrations $(0.5,1$ and $5 \mu \mathrm{M})$. The low concentration of $0.5 \mu \mathrm{M}$ did not induce any cell death in either of the neuronal cell types, while we detected differential neuronal toxicity with $1 \mu \mathrm{M}$ that induced dopaminergic cell death but not in immortalized neuronal cells. At higher concentrations, fibrils promoted neuronal cell death in both cell types. Our results suggest that human dopaminergic neurons are more susceptible to this external stimulus since neuronal death occurred at lower fibril concentrations (Fig. 8e,f). Across independently performed assays, some variability was observed, but most of the data indicate a slightly larger impact from the thinner fibrils formed at $37^{\circ} \mathrm{C}$. One indicator of this difference is also seen in the treated neurons' morphology, which is noticeably changed by the fibrils formed at $37^{\circ} \mathrm{C}$, but not by those prepared at $22^{\circ} \mathrm{C}$ (Supplementary Fig. 9). Thus, both fibril polymorphs are biologically active, with the in vitro seeding assays and cytotoxicity measurements indicating a modest, but nonetheless detectable, difference between the two polymorphs.

\section{Discussion}

We prepared amyloid-like fibrils from mutant htt exon1 that lacked undesirable modifications of its crucial htt ${ }^{\mathrm{NT}}$ segment, and studied the fibril structure and how it depends on the fibrillation temperature. At 22 and $37^{\circ} \mathrm{C}$ we obtained amyloid-like fibrils with different widths (Fig. 2), of 15 and $\sim 6 \mathrm{~nm}$, respectively. When examined by FTIR, specific differences were apparent in smaller signals present alongside the invariant dominant polyQ core signals (Fig. 2d,e). The fibrils' highly rigid polyQ domains also a

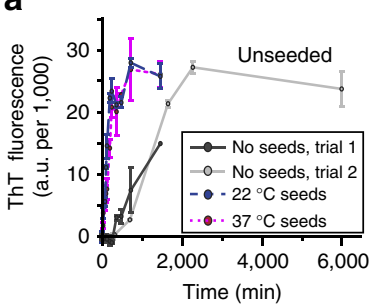

C

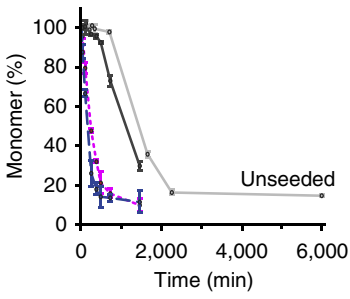

b

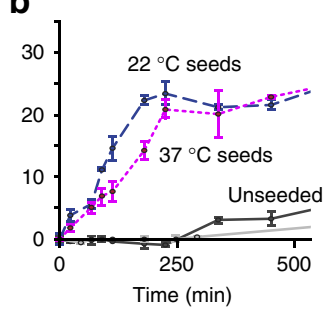

d

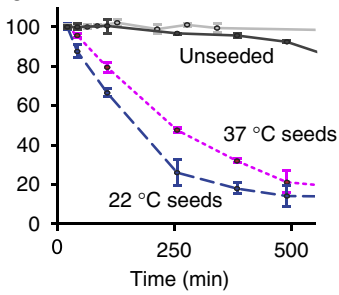

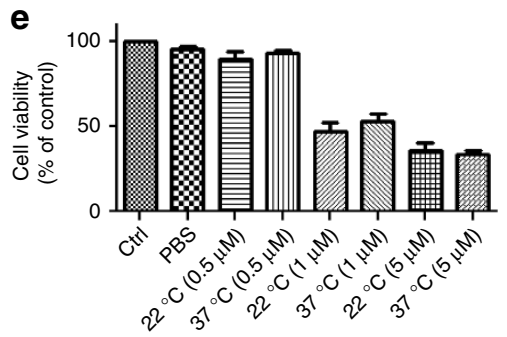

f

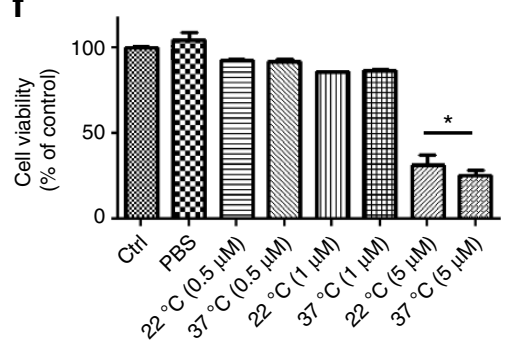

Figure 8 | PolyQ protein recruitment and neuronal toxicity assay results. (a) Aggregation kinetics at $22^{\circ} \mathrm{C}$ in the absence (solid black and grey lines) and presence (dashed lines) of pre-made seed aggregates, detected as ThT fluorescence at indicated time points after complete trypsin cleavage of the htt exon1 fusion protein. Dark blue and magenta dashed lines reflect the aggregation in presence of $20 \mathrm{~mol}-\%$ htt exon1 aggregates formed at 22 and $37^{\circ} \mathrm{C}$, respectively. The unseeded reactions have lag phases exceeding $4 \mathrm{~h}$, which are eliminated by the seeds. Error bars indicate s.d., with $n=2-3$, as described in the Methods section. (b) Enlargement of the first 500 min. (c,d) Results of a single $(n=1)$ HPLC measurement of the residual monomer concentration after aggregate sedimentation, applied to the same samples, as a complementary measure of aggregation. Error bars reflect the estimated peak integration error as described in the Methods. (e) Cellular viability of human dopaminergic neuronal cells upon exposure to varying concentrations of pre-formed fibrils prepared at 22 and $37^{\circ} \mathrm{C}$. The data reflect MTT reduction assays performed after $24 \mathrm{~h}(n=2$; two biological replicates with three technical replicates each-shown is the mean with s.d. compared to non-treated controls set at 100\%). (f) Cell viability assay data for a $24 \mathrm{~h}$ exposure of immortalized HT-22 neurons ( $n=2$; two biological replicates with 6 technical replicates each-shown is the mean with s.d. compared to non-treated controls set at $100 \%$; ${ }^{\star} P<0.05$, Mann-Whitney non-parametric test) 
featured the same characteristic ssNMR signature ${ }^{46}$. In prior work on these same htt exon 1 fibrils ${ }^{15,17}$, we used ssNMR to reveal $\sim 20$-residue-long $\beta$-strands forming a $\beta$-hairpin structure within the aggregated polyQ domain and used in silico analysis to show that alternative polyQ models have distinct ssNMR spectral signatures.

By CP-based ssNMR we observed signals from the htt ${ }^{\mathrm{NT}}$ $\alpha$-helix previously observed in HNTF peptide fibrils ${ }^{13,15,32}$, showing its presence in full-length mutant htt exon1 fibrils. The exon1 htt ${ }^{\mathrm{NT}}$ helix experiences significant dynamics that reflect molten-globule-like dynamics also seen for $\alpha$-helical htt ${ }^{\mathrm{NT}}$ in HNTF fibrils ${ }^{15}$. These dynamics reduce ssNMR peak intensities and may in part explain why previously published ssNMR spectra of mutant htt exonl fibrils failed to show signal from the htt ${ }^{\mathrm{NT}}$ (refs 15,16). It is also possible that the exon1 aggregation process was modulated by residual $\mathrm{htt}^{\mathrm{NT}}$-attached linker residues ${ }^{16}$, which are avoided in our exon1 and HNTF constructs with unmodified 17-residue $\mathrm{htt}^{\mathrm{NT}}$ segments. Our observation of $\alpha$-helical structure in the $\mathrm{htt}^{\mathrm{NT}}$ of fibrillar exon1 lends further support to the idea that $\alpha$-helical htt ${ }^{\mathrm{NT}}$ interactions play key roles in the htt exon1 aggregation mechanism. $\mathrm{Htt}^{\mathrm{NT}}$ is thought to initiate and accelerate aggregation via the formation of $\mathrm{htt}^{\mathrm{NT}}$ $\mathrm{htt}^{\mathrm{NT}} \alpha$-helical bundles ${ }^{13,25,4 \mathrm{P}}$. Flanking domain interactions play similarly important roles in the aggregation pathways of other polyQ disease proteins ${ }^{22,23}$.

The most notable ssNMR signals for the PRD are from prolines, present in both PPII and IDP-like random-coil structures. Their relative populations, estimated from directexcitation ${ }^{13} \mathrm{C}$ spectra, appear to be $2: 1$ independent of the fibril formation temperature (Supplementary Fig. 4a,b). The PPII structure is likely due to the two oligoPro segments of the PRD (Fig. 1a), of which ssNMR previously showed the first to adopt a PPII structure in HNTF fibrils ${ }^{13,15}$. The 2:1 intensity ratio shows that the remaining 10 Pro of the PRD do not form stable PPII helices. Perhaps surprisingly, this is not accompanied with IDPlike dynamics, given that the IDP-like Pro are visible in CP spectra. The PDSD and DIPSHIFT experiments indicate similar dynamics for both types of CP-detected Pro residues (Fig. 4). Interestingly, significant $\mathrm{CP}$-based signals are seen for residues up to V103. Thus, these parts of the PRD that do not occupy regular secondary structure are, nonetheless, not free to move around. We attribute this lack of motion to intermolecular interactions due to clustering of PPII- and $\alpha$-helical flanking domains ${ }^{23,54}$. Such interactions also explain the reduced binding by the MW7 antibody (Fig. 7c), while the C-terminal tail is flexible and accessible for strong MW8 binding (Fig. 7c). Thus, are data reveal a transition from a polyQ-proximal semi-rigid PRD segment to a highly dynamic flexible tail, with an apparent transition point at or near residue V103. This is sketched schematically in Fig. 9a, with the mobile C-terminal tail segments shown top right in red. The immobilized PRD segments are not as rigid as the $\beta$-sheet polyQ amyloid core, as they experience dynamics similar to those of the htt ${ }^{\mathrm{NT}} \alpha$-helices ${ }^{15}$.

The INEPT-based ssNMR spectra also contain signals from both IDP-like and PPII-helical Pro, indicating the presence of exposed and highly dynamic PPII helices in a subset of the protein molecules in the sample. The HNTF fibrils without C-terminal PRD segments lack such INEPT signals. We speculate that the first oligoPro segment is typically immobilized in its location directly attached to the immobile polyQ core (see ref. 15), while the mobile segments are in the latter part of the PRD (top right of Fig. 9a). Those exon1 monomers with the more mobile PRDs should be more accessible to interacting proteins, including the MW7 antibody (Fig. 7c).

Prior studies have indicated polymorphism in aggregates formed by htt exon1 and other N-terminal fragments, with corresponding effects on the aggregates' cellular toxicity $4,8,9,12$. Both in our hands (Fig. 2) and in earlier work ${ }^{8}$, FTIR indicated structural differences between exon1 fibrils made at different temperatures. By ssNMR we observe differences not in the polyQ domain, but rather in the other exon 1 domains. For instance, the PRD domains of $37^{\circ} \mathrm{C}$ fibrils have a larger proportion of highly flexible (Fig. 3i) and solvent-exposed (Fig. 7) residues. Conversely, Pro residues in $22^{\circ} \mathrm{C}$ fibrils are more restricted in their motion (Fig. 3) and less solvent-accessible (Fig. 7). Thus, unlike prior studies $^{8,12}$, we find the polymorphism to be predominantly reflected in the dynamics and accessibility of the non-amyloid flanking domains.

As discussed previously ${ }^{15}$, fibrillar $\mathrm{htt}^{\mathrm{NT}} \alpha$-helices are stabilized by intermolecular interactions that immobilize them enough to render them visible by CP ssNMR. These htt ${ }^{\mathrm{NT}}$ interactions are important for the oligomerization of htt exon1 and contribute to the stability of the fibrils ${ }^{32}$. We propose that in the mature fibrils flanking domain interactions similarly sequester and immobilize the PRD domains, and thus limit accessibility and binding by proteins for the most Pro-rich parts of the PRD-for example, the MW7 antibodies (Fig. 7c). The origins of this are found in the polyQ amyloid core. SSNMR studies of aggregates formed by polyQ-expanded exon 1 and polyQ peptides note that polyQ amyloid contains long antiparallel $\beta$-strands with few turn a

b
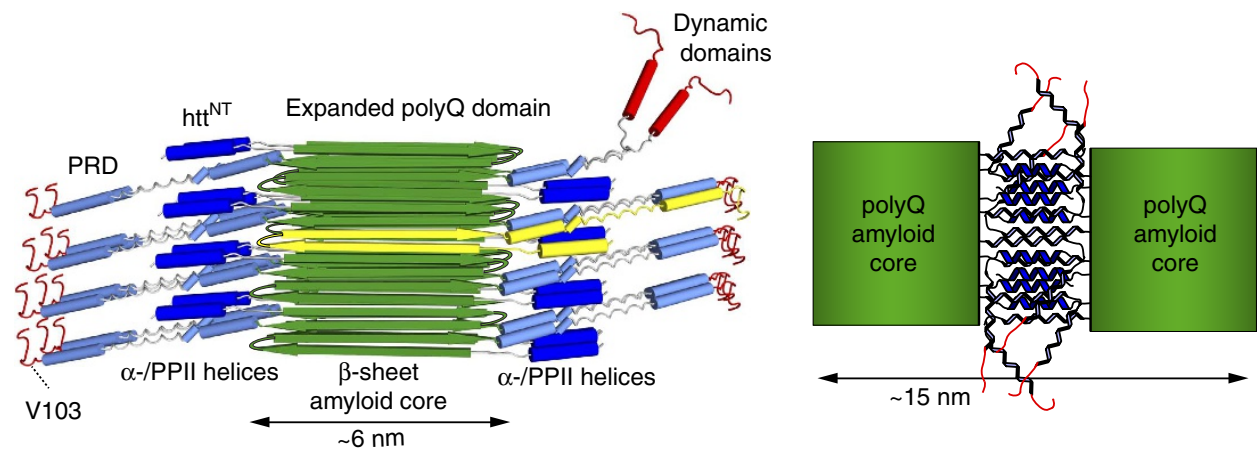

Figure 9 | Schematic proposed model of htt exon1 fibrils. (a) The htt ${ }^{\mathrm{NT}} \alpha$-helices (dark blue) and PRD PPII helices (light blue) are immobilized and tightly clustered on the perimeter of the rigid amyloid core (green $\beta$-strands). C-terminal domains show increased dynamics, either in the form of the unstructured C-terminal tail or a subpopulation of more exposed PRDs (top right; red). An individual protein monomer with its $\beta$-hairpin-based polyQ core is shown with lighter (yellow) $\beta$-strands. (b) Schematic illustration of interfilament flanking domain interactions that we propose to explain the larger TEM-based widths of the fibrils formed at $22^{\circ} \mathrm{C}$, as well as the observed differences in accessibility and immobilization of the PRD. 
regions $^{17,45}$. In our mutant htt exon 1 fibrils with 44 -residue polyQ domains, we observe $90 \%$ of the residues in the $\beta$-sheet parts of $\beta$-hairpins, separate from the $\sim 10 \%$ of polyQ residues that form the single intervening $\beta$-turn ${ }^{17}$. The intramolecular $\beta$-hairpin places flanking domains in close proximity to each other in the assembled fibril, limiting their freedom of motion and accessibility (Fig. 9). The polyQ-proximal secondary structure elements are further constrained by the short linkers that connect them to the rigid amyloid core ${ }^{13,15}$. Thus, the $\beta$ sheet fibre core is surrounded by sterically constrained and densely packed $\alpha$ - and PPII-helical flanking domains, in contrast to reports that the PRD is primarily dynamic ${ }^{14,16}$. Figure 9a shows a schematic structural model designed to illustrate the relative dimensions of the flanking domains and an amyloid core featuring 20 -residue $\beta$-strands. The latter part of the PRD is likely only weakly immobilized in a single, $\sim 6 \mathrm{~nm}$-wide, filament. We hypothesize that the structural and motional features of the $\sim 15 \mathrm{~nm}$-wide $22^{\circ} \mathrm{C}$ fibrils are most easily explained by flanking domain interactions tying together two filaments, as illustrated in Fig. 9b. This would intertwine the polyQ-proximal flanking segments of the filaments through additional interactions among the $\alpha$ - and PPII helices ${ }^{54}$. Nonetheless, as detected in PDSD and DIPSHIFT experiments, these flanking domains retain moltenglobule-like dynamics that greatly reduce the dipolar coupling constants and thus limit ssNMR sensitivity and complicate longrange distance measurements.

Both fibril polymorphs have a strong seeding ability that affects both expanded and non-expanded polyQ aggregation (Fig. 8 and Supplementary Fig. 8). Intriguingly, a small, but seemingly significant, difference is observed between the two polymorphs. This difference in seeding activity shows opposite trends for the two different target peptides, which precludes a straightforward explanation in absence of further studies. Both fibril polymorphs were also shown to have cytotoxic and morphological effects when provided extracellularly to neuronal cells, with a more visible impact by fibrils formed at $37^{\circ} \mathrm{C}$. On the basis of the available data, it remains unclear what dictates the differences in seeding ability and toxicity, and whether or how these two activities may be related. In terms of the cellular impacts, it is likely that a key determinant relates to the ability of fibrils to be taken up, which may depend on the fibril stability and width ${ }^{8,55,56}$. The exposure of $\mathrm{htt}^{\mathrm{NT}}$ and PRD domains may also be significant as they modulate interactions with cellular membranes, which may also affect cellular uptake and cytotoxic membrane disruption ${ }^{57}$.

Thus, our results point to differences in the flanking domains' exposure and interactions as being important in htt exon1 aggregates' structure and function. Factors that modulate flanking domain interactions are known to affect cellular toxicity. In htt ${ }^{\mathrm{NT}}$, these factors include covalent PTMs and non-covalent binding by chaperones and antibodies ${ }^{25,32,58}$. The intimate interactions of the polyQ-proximal PRD segments in the fibrils rationalize the finding that also PRD-binding proteins interfere with exon1 aggregation, unless they bind the disordered tail ${ }^{28-30,33,34}$. Such PRD-based aggregation-inhibiting effects are harder to reconcile with a fibril model in which the entire PRD is flexible and exposed ${ }^{14,16}$. An intriguing question is how flanking domain arrangements may affect cellular toxicity, resistance to clearance mechanisms, membrane interactions or fibrils' ability to sequester other proteins ${ }^{59,60}$. Further structural studies will be critical to gain a complete understanding of exactly how htt exon 1 aggregate structure, stability and toxicity are correlated.

\section{Methods}

Protein expression and purification. The plasmid encoding mutant htt exon 1 with 44 consecutive glutamine residues was modified from a MBP-fusion construct described previously ${ }^{40}$. A single-step deletion mutagenesis reaction using the
QuikChange II XL site-directed mutagenesis kit (Agilent Technologies, Santa Clara, CA, USA) was used to remove from the MBP-exon1 linker region 10 amino acids (ISEFGSMSTGGG), which would otherwise remain attached to the exon1 N terminus following Factor Xa cleavage. The employed primer sequences were $5^{\prime}$ CAACCTCGGGATCGAGGGAAGGATGGCGACCCTGGAAAAGCTTATG-3' and $5^{\prime}$-CATAAGCTTTTCCAGGGTCGCCATCCTTCCCTCGATCCCGAGG TTG- $3^{\prime}$. The htt exon1 construct was codon-optimized by GenScript (Piscataway, NJ), using the OptimumGene algorithm for expression in Escherichia coli (yielding 5'-AGTAGCAACAATAATAATAATAACAACAACAACAACCTGGGTATCGAAGGCCGTATGGCAACGCTGGAAAAACTGATGAAAGCATTTGAATCCCTGAAAAGTTTCCAGCAGCAACAACAACAGCAACAGCAGCAGCAGCAGCAACAGCAGCAGCAACAACAGCAGCAACAACAGCAACAGCAACAACAACAACAGCAGCAACAGCAACAACAACAGCAGCAGCAGCAACAGCAACAGCCGCCGCCGCCGCCGCCGCCGCCGCCGCCGCCGCAACTGCCGCAACCGCCGCCGCAGGCGCAACCGCTGCTGCCGCAGCCGCAGCCGCCGCCGCCGCCGCCGCCGCCGCCGCCGGGCCCGGCTGTTGCTGAAGAACCGCTGCATCGCCCGAGTGGCTCCCATCACCATCACCATCAT-3'). This construct was subcloned into the pMALc2x plasmid through the restriction digest sites EcoRI and HindIII.

The fusion protein was overexpressed in E. coli BL21(DE3)pLysS cells (Invitrogen, Grand Island, NY). Isotopic labelling followed an optimized isotopic labelling protocol ${ }^{61}$, starting by growing the cells in $11 \mathrm{LB}$ medium with ampicillin and chloramphenicol at $37^{\circ} \mathrm{C}$ and 250 r.p.m. until an optical density $\left(\mathrm{OD}_{600}\right)$ of $\sim 0.65$. The cells were pelleted at $1,677 \mathrm{~g}$ for $15 \mathrm{~min}$, resuspended in $50 \mathrm{ml} \mathrm{M} 9$ salt solution lacking nitrogen and carbon sources and then pelleted again at $1,677 \mathrm{~g}$ for $15 \mathrm{~min}$, prior to resuspension in $250 \mathrm{ml} \mathrm{M} 9$ media containing $\mathrm{U}-{ }^{13} \mathrm{C}$-D-glucose and ${ }^{15} \mathrm{~N}$-ammonium chloride (Cambridge Isotope Laboratories, Tewksbury, MA). The cells were brought to their fast growth phase by cultivating at $37^{\circ} \mathrm{C}$ for $30 \mathrm{~min}$ and 250 r.p.m. A temperature ramp from 37 to $18^{\circ} \mathrm{C}$ over $30 \mathrm{~min}$ at 250 r.p.m. was applied, followed by $30 \mathrm{~min}$ at $18^{\circ} \mathrm{C}$, to prepare for induction. Protein expression was induced by adding $0.8 \mathrm{mM}$ isopropyl $\beta$-D-thiogalactopyranoside (RPI Corp., Mt Prospect, IL), along with $0.02 \%(w / v) ~\left[{ }^{13} \mathrm{C}\right]-\mathrm{D}-$ glucose, $0.01 \%(\mathrm{w} / \mathrm{v})\left[{ }^{15} \mathrm{~N}\right]$ ammonium chloride and $100 \mu \mathrm{M} \mathrm{ZnSO}_{4}$. The fusion protein was overexpressed at $18^{\circ} \mathrm{C}$ for $16 \mathrm{~h}$, after which the cells were pelleted for $20 \mathrm{~min}$ at $7,000 \mathrm{~g}$. Cell pellets were stored at $-20^{\circ} \mathrm{C}$ at least $12 \mathrm{~h}$ before lysis. To lyse the cell, cell pellets were thawed on ice for $30 \mathrm{~min}$. Then, the pellets were resuspended in buffer A, which is PBS ( $\mathrm{pH} 7.4$ ) containing $0.02 \%(\mathrm{w} / \mathrm{w})$ sodium azide that had been sterilized by filtration through a Nalgene Rapid-Flow $500 \mathrm{ml}$ bottle top $0.2 \mu \mathrm{m}$ filter (Thermo Scientific, Waltham, MA). The resuspended cells were kept on ice, following addition of $1 \mathrm{mM}$ phenylmethanesulfonyl fluoride (ACROS, Fair Lawn, NJ), and $2 \mathrm{mg} \mathrm{ml}^{-1}$ lysozyme (Hampton Research, Aliso Viejo, CA). After $40 \mathrm{~min}$., cells were broken by sonication (Misonix Inc., Farmingdale, NY) on ice, applying $\sim 40 \mathrm{~W}$ of sonication for a total of $20 \mathrm{~min}$, alternating $10 \mathrm{~s}$ pulses and breaks of $10 \mathrm{~s}$. Cell debris was removed by centrifugation $(38,720 \mathrm{~g}$ for $1 \mathrm{~h})$. The soluble fusion protein was purified into PBS buffer over a 50-200 mM imidazole gradient using a nickel column, and then buffer-exchanged into PBS buffer to remove the residual imidazole $^{15}$. Purity and identity were verified by SDS-PAGE (12\%) and electrospray ionization time-of-flight mass spectrometry (Genomics and Proteomics Core Laboratories, University of Pittsburgh), which was also used to verify the molecular mass and isotopic labelling (where applicable) of the fusion protein, the cleaved MBP solubility tag and the htt exon1 monomer.

Fibril formation. The purified fusion protein was buffer-exchanged to buffer A (defined above) using centrifugal filter units (Millipore, Billerica, MA). To release htt exon1, the fusion protein was cleaved by treating with Factor Xa (Promega, Madison, WI) at 22 or $37^{\circ} \mathrm{C}$, as indicated. After addition of $0.55 \mu \mathrm{g}$ of Factor Xa to a $10 \mu \mathrm{l} 44.7 \mu \mathrm{M}$ solution of the fusion protein $(28.7 \mu \mathrm{g})$, the progression of cleavage and aggregation was monitored by SDS-PAGE (Bio-Rad Mini-Protein Precast TGX Gels 12\%) and TEM (see below). The full SDS-PAGE gels are also shown in Supplementary Fig. 1. Samples $(10.5 \mu \mathrm{l})$ were mixed with an equal volume of SDSPAGE loading dye to terminate the reaction, and then analysed by SDS-PAGE (Bio-Rad Mini-Protein Precast TGX Gels 12\%). For MAS ssNMR studies, uniformly ${ }^{13} \mathrm{C}$ - and ${ }^{15} \mathrm{~N}$-labelled $\left(\mathrm{U}_{-}{ }^{13} \mathrm{C},{ }^{15} \mathrm{~N}\right)$ fusion protein was cleaved and allowed to aggregate over 3 days, after which the labelled htt exonl fibrils were pelleted down at 3,000 $\mathrm{g}$ for $20 \mathrm{~min}$ and resuspended in $1 \mathrm{ml}$ buffer A. MAS ssNMR samples were packed in $3.2 \mathrm{~mm}$ MAS ssNMR sample holders (Bruker Biospin, Billerica, $\mathrm{MA}$, and CortecNet, Brooklyn, NY) using a home-built ultracentrifugal samplepacking tool ${ }^{62}$ operated for $1 \mathrm{~h}$ at $150,000 \mathrm{~g}$. The supernatant was discarded, and pelleted fibrils were washed at least three times with buffer A prior to sealing of the MAS rotor.

Transmission electron microscopy. The fibril morphology and progression of fibril formation by htt exon 1 were monitored using negative-stain TEM. Aliquots of sample were diluted with PBS buffer, and then deposited onto freshly glowdischarged carbon-coated copper grids. After removal of excess buffer, grids were treated with negative stain that was adsorbed for $30 \mathrm{~s}$ prior to blotting. The 22 and $37^{\circ} \mathrm{C}$ exon 1 fibrils were stained with $1 \%(\mathrm{w} / \mathrm{v})$ uranyl acetate and $1 \%$ phosphotungstic acid, respectively. The 22 and $37^{\circ} \mathrm{C}$ exon 1 fibrils in Supplementary Fig. 3 were stained with $1 \%(\mathrm{w} / \mathrm{v})$ uranyl acetate. Images were obtained at 6,500-30,000fold magnification using a Tecnai T12 TEM (FEI, Hillsboro, OR) operating at 
$120 \mathrm{kV}$ and equipped with an UltraScan 1000XP CCD camera (Gatan, Pleasanton, $\mathrm{CA}$ ). Fibril widths were measured using Image)'s straight line freehand tool (NIH, Bethesda, MD). Each measurement spanned the length of the negativestained area of the fibre with similar contrast. Pooled positive stain on the edges of the fibres was not included in the measurements. In images with low resolution, the fibre diameter was determined in regions with the clearest defined boundaries. At least three measurements were obtained per fibre.

FTIR spectroscopy. FTIR spectroscopy was performed using an MB series spectrophotometer with the PROTA software (ABB Bomem, Quebec City, QC, Canada). Aggregates were harvested by centrifugation for $30 \mathrm{~min}$ at $20,817 \mathrm{~g}$ in a tabletop Eppendorf 5415C centrifuge, and pellets washed three times with PBS buffer. Pellets containing aggregates were resuspended in either PBS buffer or deuterated PBS buffer at around $10 \mathrm{mg} \mathrm{ml}^{-1}$ concentration and incubated for $24 \mathrm{~h}$. Spectra of the resuspended aggregates were acquired at room temperature by placing the aggregate suspension between two polished $\mathrm{CaF}_{2}$ windows using a BioCell module (BioTools Inc.). Data from a total of 400 scans were collected with $4 \mathrm{~cm}^{-1}$ resolution at room temperature. Spectra were corrected for residual buffer absorption by subtracting the appropriate buffer-alone spectrum interactively until a flat baseline was obtained between 1,700 and $1,800 \mathrm{~cm}^{-1}$. Second-derivative spectra for the amide I region were calculated from the primary spectrum by using the PROTA software.

Dot blot antibody-binding assays. Identically sized aliquots of samples containing $1 \mu \mathrm{g}$ of unaggregated MBP-fusion protein or aggregated protein in buffer A were transferred to a nitrocellulose membrane using a Bio-Dot apparatus (Bio-Rad, \#170-6,545). Blots were incubated overnight with Odyssey Blocking Buffer (PBS) from LI-COR Biosciences (Lincoln, NE, USA), washed three times with TBST (10 mM Tris- $\mathrm{HCl}, \mathrm{pH} 7.5,150 \mathrm{mM} \mathrm{NaCl}, 0.1 \%(\mathrm{v} / \mathrm{v})$ Tween-20, 0.05\% (w/v) sodium azide) and incubated with a 1:5,000 dilution of the appropriate antibodies for $3 \mathrm{~h}$. Two independent dot blot assays were performed. The MW1, MW7 and MW8 antibodies developed in the Patterson lab ${ }^{35}$ were obtained from the Developmental Studies Hybridoma Bank (DSHB), created by the NICHD of the NIH and maintained at The University of Iowa, Department of Biology, Iowa City, IA. As controls, we obtained from the DSHB, 2A12 anti-GASP (deposited by Krasnow, M.A.; DSHB Hybridoma Product 2A12) and anti-glass-bottom boat (GBB 3D5-24; Guillermo Marquéz; University of Minnesota) antibodies against drosophila proteins. After washing with TBST to remove unbound material, blots were incubated for $2 \mathrm{~h}$ with a 1:10,000 dilution of Alexa Flour 680 conjugate of anti-mouse IgG (Invitrogen, A21057) and then washed four times with TBST. Blots were visualized using a LI-COR Odyssey Infrared Imaging System (LI-COR Biotechnology, Lincoln).

Seeding assays. The aggregates' seeding ability was measured using seeding assays $s^{21,37,50}$. As seeding material, mutant htt exon 1 fibrils were obtained after 5 days of aggregation at 22 and $37^{\circ} \mathrm{C}$, using starting monomer concentrations of $0.2 \mathrm{mg} \mathrm{ml}^{-1}(15.5 \mu \mathrm{M})$ and $0.14 \mathrm{mg} \mathrm{ml}^{-1}(10.1 \mu \mathrm{M})$, respectively. The aggregates were pelleted at $3,220 \mathrm{~g}$ for $10 \mathrm{~min}$, followed by four washing steps with PBS buffer. The final aggregates were resuspended and vortexed for $30 \mathrm{~min}$, and then sonicated for $10 \mathrm{~s}$ followed by $10 \mathrm{~s}$ of rest time for a total of $30 \mathrm{~s}$ sonication time. The freshly sonicated samples were incubated on ice for $5 \mathrm{~min}$ and then used as seeds the seeding experiments. Several complementary seeding assays were performed; the first involving a previously described htt exon1-seeding protocol ${ }^{50}$ that measures the effects of the seeds on the aggregation kinetics of our fusion protein upon trypsin cleavage. The protease cleavage was performed for $10 \mathrm{~min}$ on ice at a protease/substrate molar ratio of 1:3, after which the reaction was quenched with phenylmethylsulfonyl fluoride inhibitor/substrate molar ratio of 150:1. The reaction mixture was split into three samples. To two of the samples $20 \mathrm{~mol}-\%$ of pre-aggregated seeds were added. Next, the volume of all samples was adjusted with PBS to obtain a polyQ monomer concentration of $11.6 \mu \mathrm{M}$, after which aggregation was done at $22^{\circ} \mathrm{C}$. For each sample the aggregation progression was monitored using a combination of ThT assays (in duplicate or triplicate; see below) and a complementary HPLC-based assay, which detect the aggregated and monomer protein, respectively. These methods are described below. Complementary seeding assays probed the effect of the 20 mol- $\%$ of the exon 1 fibril seeds on the aggregation at $37^{\circ} \mathrm{C}$ of $h t t^{\mathrm{NT}} \mathrm{Q}_{23} \mathrm{P}_{10} \mathrm{~K}_{2}$ peptide. The $\mathrm{htt} \mathrm{t}^{\mathrm{NT}} \mathrm{Q}_{23} \mathrm{P}_{10} \mathrm{~K}_{2}$ was prepared and disaggregated ${ }^{21}$ in 1:1 (v/v) mixture of trifluoroacetic acid (TFA) and hexafluoroisopropanol overnight. The buffer was evaporated off under a $\mathrm{N}_{2}$ stream and the peptide was dried under vacuum for $1 \mathrm{~h}$. The residue was dissolved in $\mathrm{H}_{2} \mathrm{O}$ adjusted to $\mathrm{pH} 3$ with TFA. Residual aggregates were removed by

ultracentrifugation at $386,000 \mathrm{~g}$ for $1 \mathrm{~h}$, after which the $\mathrm{pH}$ was adjusted to $7.0 \mathrm{using}$ $10 \times$ PBS buffer that was subsequently diluted 10 -fold. Next, the seeds were added and the reaction kinetics monitored (in presence and absence of seeds) using ThT and HPLC-based assays, described below.

ThT fluorescence assays. Aggregates were resuspended by aspiration and aliquots were diluted into a ThT stock solution $(5 \mu \mathrm{M}$ ThT, $10 \mathrm{mM}$ sodium phosphate, $150 \mathrm{mM} \mathrm{NaCl}, \mathrm{pH} 7.0$ ). Samples were excited at $445 \mathrm{~nm}$ and the emission was recorded at $489 \mathrm{~nm}$ over several seconds on a FluoroMax-4 spectrofluorometer
(Horiba; Kyoto, Japan). The excitation and emission slits were 2 and $4 \mathrm{~nm}$, respectively. The ThT assays on the seeded aggregation at $22{ }^{\circ} \mathrm{C}$ starting with the htt exon 1 fusion protein were performed in duplicate, except for the following measurements performed in triplicate: $22^{\circ} \mathrm{C}$ seeds: $15,45,75,105,195$ and $735 \mathrm{~min}$.; $37^{\circ} \mathrm{C}$ seeds: $15,45,75$ and $135 \mathrm{~min}$. The corresponding ThT measurements of the unseeded aggregation were performed in duplicate, except for trial 1's first four points (which were measured in triplicate) and its final time point that was measured once. The ThT assays of the seeded aggregation at $37^{\circ} \mathrm{C}$ of the $\mathrm{htt}{ }^{\mathrm{NT}} \mathrm{Q}_{23} \mathrm{P}_{10} \mathrm{~K}_{2}$ peptide were based on duplicate measurements, except for triplicate measurements of the $1,200,1,740$ and 2,700 min time points $\left(37^{\circ} \mathrm{C}\right.$ seeds), and single measurements at $60 \mathrm{~min}$ (both seeds). The corresponding ThT assays of the unseeded material were performed in duplicate, except for 2,700 min time point that was measured in triplicate and the $60 \mathrm{~min}$ point that was measured once.

HPLC-based sedimentation assay. As a complementary assay to the ThT assays of seeded and unseeded aggregation, we also performed a single measurement each of the peptide monomer concentration using an established HPLC-based sedimentation assay ${ }^{21,63}$. To do so, an aliquot was removed from the reaction mixtures at the indicated time points, and the solid material was pelleted at $20,800 \mathrm{~g}$ for $15 \mathrm{~min}$. The supernatant was diluted $2 \times$ in formic acid, and loaded on an Agilent Zorbax SB-C8 $4.6 \times 50 \mathrm{~mm}$ column $(1.8 \mu \mathrm{m}$ particle size $)$ using an analytical HPLC (Agilent Technologies). The monomer was eluted over a $15-35 \%$ gradient of acetonitrile in water, with $0.05 \%$ TFA at $37^{\circ} \mathrm{C}$. The elution of the monomer species was monitored by absorbance at $215 \mathrm{~nm}\left(\mathrm{~A}_{215}\right)$, as it has no significant absorbance at $\mathrm{A}_{280}$. The relative amount of monomer in each sample was determined by integration of $A_{215}$ peaks using the manual integration analysis mode with a manually defined linear baseline correction as provided by the ChemStation for the LC systems software (Agilent Technologies). To estimate the error in this manual integration and baseline correction, three independent integrations were applied to each datapoint, yielding an approximate measurement error of $5 \%$ in most of the measured values (see individual error bars in Fig. 8).

Neuronal toxicity assays. $\mathrm{Htt}$ exonl aggregates were prepared at 22 and $37^{\circ} \mathrm{C}$, as described above, at concentrations of 14.5 and $10.1 \mu \mathrm{M}$, respectively. The resulting fibrils were examined by negative stain TEM to verify the generation of the wide and narrow fibril polymorphs. After 1 week, the aggregated material was resuspended by aspiration followed by vortexing and shaking for $30 \mathrm{~min}$. The remaining material was pelleted at $3,220 \mathrm{~g}$ for $10 \mathrm{~min}$, and the soluble MBP was removed by buffer exchange three times into PBS $(0.02 \% \mathrm{w} / \mathrm{v} \mathrm{NaN})$. After the samples were thoroughly resuspended, they were sonicated as described above. Before use in the toxicity assays, the fibrils were washed three times with PBS buffer and sonicated. The employed LUHMES cells are human-differentiated neurons derived from a clone of MESC2.10 cells. These human dopaminergic neurons were extensively studied and validated previously ${ }^{51-53,64}$. The employed cells were kindly provided to us by Professor Marcel Leist (University of Konstanz, Germany), and were used without further authentication. The cells were tested for mycoplasma contamination and were found to be mycoplasma-free. Non-differentiated LUHMES cells were grown in coated plates with poly-L-lysine $\left(0.1 \mathrm{mg} \mathrm{ml}^{-1}\right)$ in growth medium (DMEM/F12 medium supplemented with $1 \%$ N2-supplement (Life Technologies, Carlsbad, CA, USA), $100 \mathrm{U} \mathrm{ml}^{-1}$ penicillin, $100 \mathrm{mg} \mathrm{ml}^{-1}$ streptomycin and $0.04 \mu \mathrm{g} \mathrm{ml}{ }^{-1}$ basic fibroblast growth factor (R\&D Systems, Minneapolis, MN, USA)). For the differentiation experiments, LUHMES cells were grown in coated plates with poly-L-lysine $\left(10 \mu \mathrm{g} \mathrm{ml}^{-1}\right)$ followed by fibronectin $\left(5 \mu \mathrm{g} \mathrm{ml}^{-1}\right)$. Dopaminergic neurons were differentiated in DMEM/F12 medium with $1 \% \mathrm{~N} 2$-supplement, $1 \mu \mathrm{g} \mathrm{ml}^{-1}$ tetracycline, $100 \mathrm{U} \mathrm{ml}^{-1}$ penicillin, $100 \mathrm{mg} \mathrm{ml}^{-1}$ streptomycin, $0.49 \mathrm{mg} \mathrm{ml}^{-1}$ dibutyril cyclic AMP (Sigma-Aldrich) and $2 \mathrm{ng} \mathrm{ml}^{-1}$ glial cell-derived neurotrophic factor ${ }^{52,53}$. Following 6-7 days of in vitro differentiation, LUHMES cells expressed the dopamine transporter, the vesicular monoamine transporter 2 , tyrosine hydroxylase and the neuronal form of $\beta$-III tubulin ${ }^{53,65}$. The immortalized mouse hippocampal HT-22 cells were cultured in Dulbecco's modified Eagle's medium with the addition of $10 \%$ heat-inactivated fetal calf serum, $100 \mathrm{U} \mathrm{ml}^{-1}$ penicillin, $100 \mathrm{mg} \mathrm{ml}^{-1}$ streptomycin and $2 \mathrm{mM}$ glutamine. Toxicity measurements were performed by administering the sonicated fibrils at the indicated protein concentrations within the growth medium. After 24 and $48 \mathrm{~h}$ of treatment, quantification of cell viability was performed via a MTT (3-(4,5-dimethyl-2-thiazolyl)-2,5-diphenyl-2H-tetrazolium bromide) reduction assay at $0.5 \mathrm{mg} \mathrm{ml}^{-1}$ for $1 \mathrm{~h}$. The reaction was terminated by removing the MTT solution and freezing the plate at $-20^{\circ} \mathrm{C}$ for at least $1 \mathrm{~h}$. DMSO solvent was added to each well for $30 \mathrm{~min}$ under shaking conditions at $37^{\circ} \mathrm{C}$. The absorbance of each well was determined with a Synergy H1 Multi-Mode reader (Biotek, LA) at $570 \mathrm{~nm}$ with a reference filter at $630 \mathrm{~nm}$ (refs 52,53).

MAS ssNMR spectroscopy. Unless specified otherwise, MAS ssNMR experiments were performed using a wide-bore Bruker Avance I NMR spectrometer operating at ${ }^{1} \mathrm{H}$ Larmor frequency of $600 \mathrm{MHz}$ (14.1 Tesla) and triple-channel (HCN) Bruker 3.2 mm MAS NMR probes. Isotopically labelled samples were prepared and packed into $3.2 \mathrm{~mm}$ MAS rotors using an ultracentrifugal packing device ${ }^{15,62}$. CP-based peak assignments were obtained using $2 \mathrm{D}^{13} \mathrm{C}-{ }^{13} \mathrm{C}$ spectra employing DARR $^{66}$ mixing, as well as standard heteronuclear 2D MAS ssNMR 
assignment spectra. Dynamics were probed via dipolar-recoupling curves based on a series of 2D PDSD experiments, with mixing times ranging from $0,15,50,100$, 250 to $500 \mathrm{~ms}$. 2D peak volumes were integrated using the Gaussian peak fitting routines of the Sparky NMR software package, and normalized relative to corresponding diagonal peak volumes at zero mixing. The errors in the measured peak intensity were estimated based on the noise peak intensities in the spectra. ${ }^{1} \mathrm{H}-{ }^{13} \mathrm{C}$ dipolar couplings were probed via DIPSHIFT experiments ${ }^{49}$ using a 3.2-mm triple-channel HCN MAS probe in a wide-bore $750 \mathrm{MHz}$ spectrometer from Bruker Biospin acquired via NIH S10 grant OD012213-01. The DIPSHIFT experiments employed a R18 $8_{1}^{7}$ pulse sequence ${ }^{67,68}$, at $10 \mathrm{kHz}$ MAS and $277 \mathrm{~K}$. We measured 12 increments constituting a $100 \mu$ s rotor period each, up to a maximum recoupling time of $1.1 \mathrm{~ms} .{ }^{1} \mathrm{H}-{ }^{13} \mathrm{C}$ dipolar recoupling in the DIPSHIFT experiment was enabled by application of a $\mathrm{R} 18_{1}^{7}$ pulse sequence on the ${ }^{1} \mathrm{H}$ channel at a $91 \mathrm{kHz}$ $\mathrm{RF}$ power level. The initial ${ }^{13} \mathrm{C}$ signal was generated with $\mathrm{CP}$, using a $1.5 \mathrm{~ms}$ contact time. Highly mobile segments of the aggregated exon 1 were identified using scalarbased spectroscopy employing refocused INEPT ${ }^{1} \mathrm{H}-{ }^{13} \mathrm{C}$ transfers combined with ${ }^{13} \mathrm{C}-{ }^{13} \mathrm{C}$ transfers using $\mathrm{P}^{1}{ }_{3}$ TOBSY ${ }^{69-71}$. Water-exposure measurements were performed using ssNMR experiments in which rigid ${ }^{1} \mathrm{H}$ signals were suppressed by $\mathrm{T}_{2}$ relaxation filtering, after which ${ }^{1} \mathrm{H}-{ }^{1} \mathrm{H}$ diffusion facilitated transfer of the remaining polarization of mobile solvent protons back into the immobilized protein assemblies ${ }^{13,15}$. The resulting polarization buildup in the protein residues was then monitored via $1 \mathrm{D}^{1} \mathrm{H}_{-}{ }^{13} \mathrm{C} C \mathrm{CP}$ spectroscopy. $2 \mathrm{D}^{1} \mathrm{H}^{13} \mathrm{C}$ spectra were used to verify the origin of the (mobile) ${ }^{1} \mathrm{H}$ polarization being the aqueous solvent. $\mathrm{T}_{2}$-filtered $2 \mathrm{D}{ }^{13} \mathrm{C}-{ }^{13} \mathrm{C}$ DARR spectra were used to verify the identity of dominant peaks in the $\mathrm{T}_{2}$-filted $1 \mathrm{D}$ spectra.

Experimental details for all spectra are listed in Supplementary Table $2 .{ }^{1} \mathrm{H}$ decoupling during acquisition and evolution periods was done with two-pulse phase modulation ${ }^{72}$, and MAS spinning rates were typically between 8.3 and $13 \mathrm{kHz}$ (see Supplementary Table 2). Spectra were acquired using the Bruker Topspin software, processed using NMRPipe ${ }^{73}$. Chemical shifts were assigned and analysed using the Sparky and CcpNmr Analysis software packages ${ }^{74}$. Peak intensities were measured in the Bruker's Topspin software and CcpNmr Analysis, with the error in the intensities evaluated based on the noise peaks present in empty spectral regions. Numerical simulations of the DIPSHIFT experiments were performed with the SpinEvolution programme ${ }^{15,75}$. Chemical shift referencing to 4,4-dimethyl-4-silapentane-1-sulfonic acid (for ${ }^{13} \mathrm{C}$ ) was performed by indirect referencing via the ${ }^{13} \mathrm{C}$ signals of adamantane ${ }^{15}$. Secondary shift calculations were done using published random coil shifts ${ }^{76}$.

Data availability. Chemical shifts of the synthetic HNTF peptide fibrils $\left(h_{t t}{ }^{\mathrm{NT}} \mathrm{Q}_{30} \mathrm{P}_{10} \mathrm{~K}_{2}\right)$ were reported previously ${ }^{15}$ and are accessible in the Biological Magnetic Resonance Data Bank (BMRB) as entry 25146. Assigned shifts of the mutant htt exon1 fibrils are available in Supplementary Table 3, and in the BMRB as entry 27045 . UniProt entry P42858 has been used in this study. All other data are available from the corresponding author upon reasonable request.

\section{References}

1. Bates, G. P. et al. Huntington disease. Nat. Rev. Dis. Primers 1, 15005 (2015).

2. Arrasate, M., Mitra, S., Schweitzer, E. S., Segal, M. R. \& Finkbeiner, S. Inclusion body formation reduces levels of mutant huntingtin and the risk of neuronal death. Nature 431, 805-810 (2004).

3. Sahl, S. J., Weiss, L. E., Duim, W. C., Frydman, J. \& Moerner, W. E. Cellular inclusion bodies of mutant huntingtin exon 1 obscure small fibrillar aggregate species. Sci. Rep. 2, 895 (2012).

4. Duim, W. C., Jiang, Y., Shen, K., Frydman, J. \& Moerner, W. E. Superresolution fluorescence of huntingtin reveals growth of globular species into short fibers and coexistence of distinct aggregates. ACS Chem. Biol. 9, 2767-2778 (2014).

5. Sahl, S. J. et al. Delayed emergence of subdiffraction-sized mutant huntingtin fibrils following inclusion body formation. Quart. Rev. Biophys. 49, 1-13 (2015).

6. Kodali, R. B., Williams, A. D., Chemuru, S. \& Wetzel, R. A $\beta(1-40)$ forms five distinct amyloid structures whose $\beta$-sheet contents and fibril stabilities are correlated. J. Mol. Biol. 401, 503-517 (2010).

7. Tycko, R. Physical and structural basis for polymorphism in amyloid fibrils. Protein Sci. 23, 1528-1539 (2014)

8. Nekooki-Machida, Y. et al. Distinct conformations of in vitro and in vivo amyloids of huntingtin-exon1 show different cytotoxicity. Proc. Natl Acad. Sci. USA 106, 9679-9684 (2009).

9. Sun, C.-S. et al. Conformational switch of polyglutamine-expanded huntingtin into benign aggregates leads to neuroprotective effect. Sci. Rep. 5, 14992 (2015).

10. van Ham, T. J. et al. Identification of MOAG-4/SERF as a regulator of agerelated proteotoxicity. Cell 142, 601-612 (2010).

11. Falsone, S. F. et al. SERF protein is a direct modifier of amyloid fiber assembly. Cell Rep. 2, 358-371 (2012).

12. Perevozchikova, T., Stanley, C. B., McWilliams-Koeppen, H. P., Rowe, E. L. \& Berthelier, V. Investigating the structural impact of the glutamine repeat in huntingtin assembly. Biophys. J. 107, 411-421 (2014).
13. Sivanandam, V. N. et al. The aggregation-enhancing huntingtin N-terminus is helical in amyloid fibrils. J. Am. Chem. Soc. 133, 4558-4566 (2011).

14. Bugg, C. W., Isas, J. M., Fischer, T., Patterson, P. H. \& Langen, R. Structural features and domain organization of huntingtin fibrils. J. Biol. Chem. 287, 31739-31746 (2012).

15. Hoop, C. L. et al. Polyglutamine amyloid core boundaries and flanking domain dynamics in huntingtin fragment fibrils determined by solid-state nuclear magnetic resonance. Biochemistry 53, 6653-6666 (2014).

16. Isas, J. M., Langen, R. \& Siemer, A. B. Solid-state NMR on the static and dynamic domains of huntingtin exon-1 fibrils. Biochemistry 54, 3942-3949 (2015).

17. Hoop, C. L. et al. Huntingtin exon 1 fibrils feature an interdigitated $\beta$-hairpinbased polyglutamine core. Proc. Natl Acad. Sci. USA 113, 1546-1551 (2016).

18. Duennwald, M. L., Jagadish, S., Muchowski, P. J. \& Lindquist, S. L. Flanking sequences profoundly alter polyglutamine toxicity in yeast. Proc. Natl Acad. Sci. USA 103, 11045-11050 (2006).

19. Dehay, B. \& Bertolotti, A. Critical role of the proline-rich region in huntingtin for aggregation and cytotoxicity in yeast. J. Biol. Chem. 281, 35608-35615 (2006).

20. Atwal, R. S. et al. Huntingtin has a membrane association signal that can modulate huntingtin aggregation, nuclear entry and toxicity. Hum. Mol. Genet. 16, 2600-2615 (2007).

21. Thakur, A. K. et al. Polyglutamine disruption of the huntingtin exon $1 \mathrm{~N}$ terminus triggers a complex aggregation mechanism. Nat. Struct. Mol. Biol. 16, 380-389 (2009).

22. Ellisdon, A. M., Thomas, B. \& Bottomley, S. P. The two-stage pathway of ataxin-3 fibrillogenesis involves a polyglutamine-independent step. J. Biol. Chem. 281, 16888-16896 (2006).

23. Zboray, L. et al. Preventing the androgen receptor N/C Interaction delays disease onset in a mouse model of SBMA. Cell Rep. 13, 2312-2323 (2015).

24. Bhattacharyya, A. M. et al. Oligoproline effects on polyglutamine conformation and aggregation. J. Mol. Biol. 355, 524-535 (2006).

25. Tam, S. et al. The chaperonin TRiC blocks a huntingtin sequence element that promotes the conformational switch to aggregation. Nat. Struct. Mol. Biol. 16, 1279-1285 (2009).

26. Choudhury, K. R. \& Bhattacharyya, N. P. Chaperone protein HYPK interacts with the first 17 amino acid region of huntingtin and modulates mutant HTT-mediated aggregation and cytotoxicity. Biochem. Biophys. Res. Commun. 456, 66-73 (2015).

27. Monsellier, E., Redeker, V., Ruiz-Arlandis, G., Bousset, L. \& Melki, R. Molecular interaction between the chaperone Hsc70 and the $\mathrm{N}$-terminal flank of huntingtin exon 1 modulates aggregation. J. Biol. Chem. 290, 2560-2576 (2015).

28. Khoshnan, A., Ko, J. \& Patterson, P. H. Effects of intracellular expression of anti-huntingtin antibodies of various specificities on mutant huntingtin aggregation and toxicity. Proc. Natl Acad. Sci. USA 99, 1002-1007 (2002)

29. Southwell, A. L. et al. Intrabodies binding the proline-rich domains of mutant huntingtin increase its turnover and reduce neurotoxicity. J. Neurosci. 28, 9013-9020 (2008).

30. Legleiter, J. et al. Monoclonal antibodies recognize distinct conformational epitopes formed by polyglutamine in a mutant huntingtin fragment. J. Biol. Chem. 284, 21647-21658 (2009).

31. Ehrnhoefer, D. E., Sutton, L. \& Hayden, M. R. Small changes, big impact: posttranslational modifications and function of huntingtin in Huntington disease. Neuroscientist 17, 475-492 (2011).

32. Mishra, R. et al. Serine phosphorylation suppresses huntingtin amyloid accumulation by altering protein aggregation properties. J. Mol. Biol. 424, 1-14 (2012).

33. Wang, C. E. et al. Suppression of neuropil aggregates and neurological symptoms by an intracellular antibody implicates the cytoplasmic toxicity of mutant huntingtin. J. Cell Biol. 181, 803-816 (2008).

34. Chow, W. N. V., Luk, H. W., Chan, H. Y. E. \& Lau, K.-F. Degradation of mutant huntingtin via the ubiquitin/proteasome system is modulated by FE65. Biochem. J. 443, 681-689 (2012).

35. Ko, J., Ou, S. \& Patterson, P. H. New anti-huntingtin monoclonal antibodies: implications for huntingtin conformation and its binding proteins. Brain Res. Bull. 56, 319-329 (2001).

36. Meier, B. H. \& Böckmann, A. The structure of fibrils from 'misfolded' proteins. Curr. Opin. Struc. Biol. 30, 43-49 (2014).

37. Sahoo, B., Singer, D., Kodali, R., Zuchner, T. \& Wetzel, R. Aggregation behavior of chemically synthesized, full-length huntingtin exon1. Biochemistry 53, $3897-$ 3907 (2014).

38. Scherzinger, E. et al. Huntingtin-encoded polyglutamine expansions form amyloid-like protein aggregates in vitro and in vivo. Cell 90, 549-558 (1997).

39. Bennett, M. J. et al. A linear lattice model for polyglutamine in CAG-expansion diseases. Proc. Natl Acad. Sci. USA 99, 11634-11639 (2002).

40. Poirier, M. A. et al. Huntingtin spheroids and protofibrils as precursors in polyglutamine fibrilization. J. Biol. Chem. 277, 41032-41037 (2002). 
41. Jayaraman, M. et al. Slow amyloid nucleation via $\alpha$-helix-rich oligomeric intermediates in short polyglutamine-containing huntingtin fragments. J. Mol. Biol. 415, 881-899 (2012).

42. Ruggeri, F. S. et al. Nanoscale studies link amyloid maturity with polyglutamine diseases onset. Sci. Rep. 6, 31155 (2016).

43. Farrell, H. M., Qi, P. X., Wickham, E. D. \& Unruh, J. J. Secondary structural studies of bovine caseins: structure and temperature dependence of $\beta$-casein phosphopeptide (1-25) as analyzed by circular dichroism, FTIR spectroscopy, and analytical ultracentrifugation. J. Protein Chem. 21, 307-321 (2002).

44. Tooke, L., Duitch, L., Measey, T. J. \& Schweitzer-Stenner, R. Kinetics of the selfaggregation and film formation of poly-L-proline at high temperatures explored by circular dichroism spectroscopy. Biopolymers 93, 451-457 (2010).

45. Schneider, R. et al. Structural characterization of polyglutamine fibrils by solidstate NMR spectroscopy. J. Mol. Biol. 412, 121-136 (2011).

46. Kar, K. et al. $\beta$-hairpin-mediated nucleation of polyglutamine amyloid formation. J. Mol. Biol. 425, 1183-1197 (2013).

47. Tamiola, K., Acar, B. \& Mulder, F. A. A. Sequence-specific random coil chemical shifts of intrinsically disordered proteins. J. Am. Chem. Soc. 132, 18000-18003 (2010)

48. Li, J. \& Van der Wel, P. C. A. Spinning-rate encoded chemical shift correlations from rotational resonance solid-state NMR experiments. J. Magn. Reson. 230, 117-124 (2013)

49. Munowitz, M., Griffin, R. G., Bodenhausen, G. \& Huang, T. H. Twodimensional rotational spin-echo nuclear magnetic resonance in solids: correlation of chemical shift and dipolar interactions. J. Am. Chem. Soc. 103, 2529-2533 (1981)

50. Scherzinger, E. et al. Self-assembly of polyglutamine-containing huntingtin fragments into amyloid-like fibrils: implications for Huntington's disease pathology. Proc. Natl Acad. Sci. USA 96, 4604-4609 (1999).

51. Lotharius, J. et al. Effect of mutant $\alpha$-synuclein on dopamine homeostasis in a new human mesencephalic cell line. J. Biol. Chem. 277, 38884-38894 (2002).

52. Dolga, A. M. et al. Subcellular expression and neuroprotective effects of SK channels in human dopaminergic neurons. Cell Death Dis. 5, e999 (2014).

53. Höllerhage, M. et al. Trifluoperazine rescues human dopaminergic cells from wild-type $\alpha$-synuclein-induced toxicity. Neurobiol. Aging 35, 1700-1711 (2014).

54. Larson, M. R. et al. Elongated fibrillar structure of a streptococcal adhesin assembled by the high-affinity association of $\alpha$ - and PPII-helices. Proc. Natl Acad. Sci. USA 107, 5983-5988 (2010).

55. Yang, W., Dunlap, J. R., Andrews, R. B. \& Wetzel, R. Aggregated polyglutamine peptides delivered to nuclei are toxic to mammalian cells. Hum. Mol. Genet. 11, 2905-2917 (2002).

56. Pieri, L., Madiona, K., Bousset, L. \& Melki, R. Fibrillar $\alpha$-synuclein and huntingtin exon 1 assemblies are toxic to the cells. Biophys. J. 102, 2894-2905 (2012).

57. Burke, K. A., Kauffman, K. J., Umbaugh, C. S., Frey, S. L. \& Legleiter, J. The interaction of polyglutamine peptides with lipid membranes is regulated by flanking sequences associated with huntingtin. J. Biol. Chem. 288, 14993-15005 (2013).

58. De Genst, E. et al. Structure of a single-chain Fv bound to the $17 \mathrm{~N}$-terminal residues of huntingtin provides insights into pathogenic amyloid formation and suppression. J. Mol. Biol. 427, 2166-2178 (2015).

59. Steffan, J. S. et al. The Huntington's disease protein interacts with p53 and CREB-binding protein and represses transcription. Proc. Natl Acad. Sci. USA 97, 6763-6768 (2000)

60. Yu, A. et al. Protein aggregation can inhibit clathrin-mediated endocytosis by chaperone competition. Proc. Natl Acad. Sci. USA 111, E1481-E1490 (2014).

61. Marley, J., Lu, M. \& Bracken, C. A method for efficient isotopic labeling of recombinant proteins. J. Biomol. NMR 20, 71-75 (2001).

62. Mandal, A., Boatz, J. C., Wheeler, T. \& Van der Wel, P. C. A. On the use of ultracentrifugal devices for routine sample preparation in biomolecular magicangle-spinning NMR. J. Biomol. NMR 67, 165-178 (2017).

63. O'Nuallain, B. et al. Kinetics and thermodynamics of amyloid assembly using a high-performance liquid chromatography-based sedimentation assay. Methods Enzymol. 413, 34-74 (2006).

64. Lotharius, J. et al. Progressive degeneration of human mesencephalic neuronderived cells triggered by dopamine-dependent oxidative stress is dependent on the mixed-lineage kinase pathway. J. Neurosci. 25, 6329-6342 (2005).

65. Schildknecht, S. et al. Requirement of a dopaminergic neuronal phenotype for toxicity of low concentrations of 1-methyl-4-phenylpyridinium to human cells. Toxicol. Appl. Pharmacol. 241, 23-35 (2009).

66. Takegoshi, K., Nakamura, S. \& Terao, T. ${ }^{13} \mathrm{C}-{ }^{1} \mathrm{H}$ dipolar-assisted rotational resonance in magic-angle spinning NMR. Chem. Phys. Lett. 344, 631-637 (2001).
67. Carravetta, M., Edén, M., Zhao, X., Brinkmann, A. \& Levitt, M. H. Symmetry principles for the design of radiofrequency pulse sequences in the nuclear magnetic resonance of rotating solids. Chem. Phys. Lett. 321, 205-215 (2000).

68. Zhao, X., Edén, M. \& Levitt, M. H. Recoupling of heteronuclear dipolar interactions in solid-state NMR using symmetry-based pulse sequences. Chem. Phys. Lett. 342, 353-361 (2001).

69. Morris, G. A. \& Freeman, R. Enhancement of nuclear magnetic resonance signals by polarization transfer. J. Am. Chem. Soc. 101, 760-762 (1979)

70. Baldus, M. \& Meier, B. H. Total correlation spectroscopy in the solid state. The use of scalar couplings to determine the through-bond connectivity. J. Magn. Reson. A 121, 65-69 (1996).

71. Andronesi, O. C. et al. Determination of membrane protein structure and dynamics by magic-angle-spinning solid-state NMR spectroscopy. J. Am. Chem. Soc. 127, 12965-12974 (2005).

72. Bennett, A. E., Rienstra, C. M., Auger, M., Lakshmi, K. V. \& Griffin, R. G. Heteronuclear decoupling in rotating solids. J. Chem. Phys. 103, 6951-6958 (1995).

73. Delaglio, F. et al. NMRPipe: a multidimensional spectral processing system based on UNIX pipes. J. Biomol. NMR 6, 277-293 (1995).

74. Stevens, T. J. et al. A software framework for analysing solid-state MAS NMR data. J. Biomol. NMR 51, 437-447 (2011).

75. Veshtort, M. \& Griffin, R. G. SPINEVOLUTION: a powerful tool for the simulation of solid and liquid state NMR experiments. J. Magn. Reson. 178, 248-282 (2006).

76. Zhang, H., Neal, S. \& Wishart, D. S. RefDB: a database of uniformly referenced protein chemical shifts. J. Biomol. NMR 25, 173-195 (2003).

\section{Acknowledgements}

We thank Michael Delk, Dr Cody Hoop, Dr Kenneth Drombosky and Carmen IJsebaar for technical assistance and helpful discussions, and Drs James Conway and Alexander Makhov for use of the electron microscopy facility. We acknowledge funding from the University of Pittsburgh and National Institutes of Health (NIH grants R01 GM112678 and AG019322 to P.C.A.v.d.W. and R.W., R01 GM099718 to R.W., T32 GM088119 to J.C.B.), NIH instrument grant S10 OD012213-01 and grant UL1 RR024153 from the National Center for Research Resources (NCRR). A.M.D. is supported by a Rosalind Franklin Fellowship co-funded by European Union and University of Groningen. J.C.B acknowledges support by the Achievement Rewards for College Scientists (ARCS) Foundation. Molecular graphics were prepared with UCSF Chimera, developed by the Resource for Biocomputing, Visualization, and Informatics at the University of California, San Francisco (supported by NIGMS P41-GM103311).

\section{Author contributions}

H.-K.L., P.C.A.v.d.W., J.C.B., Z.H., R.K. and I.E.K. conducted experiments. R.W., R.K., A.M.D., M.A.P. and P.C.A.v.d.W. designed experiments; P.C.A.v.d.W. and H.-K.L. wrote the paper.

\section{Additional information}

Supplementary Information accompanies this paper at http://www.nature.com/ naturecommunications

Competing interests: The authors declare no competing financial interests.

Reprints and permission information is available online at http://npg.nature.com/ reprintsandpermissions/

How to cite this article: Lin, H.-K. et al. Fibril polymorphism affects immobilized non-amyloid flanking domains of huntingtin exonl rather than its polyglutamine core. Nat. Commun. 8, 15462 doi: 10.1038/ncomms15462 (2017).

Publisher's note: Springer Nature remains neutral with regard to jurisdictional claims in published maps and institutional affiliations.

This work is licensed under a Creative Commons Attribution 4.0 International License. The images or other third party material in this article are included in the article's Creative Commons license, unless indicated otherwise in the credit line; if the material is not included under the Creative Commons license, users will need to obtain permission from the license holder to reproduce the material To view a copy of this license, visit http://creativecommons.org/licenses/by/4.0/

C The Author(s) 2017 